\title{
Quasistationary solutions of self-gravitating scalar fields around collapsing stars
}

\author{
Nicolas Sanchis-Gual, ${ }^{1}$ Juan Carlos Degollado, ${ }^{2}$ Pedro J. Montero, ${ }^{3}$ José A. Font, ${ }^{1,4}$ and Vassilios Mewes ${ }^{1}$ \\ ${ }^{1}$ Departamento de Astronomía y Astrofísica, Universitat de València, \\ Dr. Moliner 50, 46100, Burjassot (València), Spain \\ ${ }^{2}$ Departamento de Ciencias Computacionales, Centro Universitario \\ de Ciencias Exactas e Ingeniería, Universidad de Guadalajara \\ Av. Revolución 1500, Colonia Olímpica C.P. 44430, Guadalajara, Jalisco, Mexico \\ ${ }^{3}$ Max-Planck-Institute für Astrophysik, Karl-Schwarzschild-Str. 1, 85748, Garching bei München, Germany \\ ${ }^{4}$ Observatori Astronòmic, Universitat de València, \\ C/ Catedrático José Beltrán 2, 46980, Paterna (València), Spain
}

(Dated: July 2015)

\begin{abstract}
Recent work has shown that scalar fields around black holes can form long-lived, quasistationary configurations surviving for cosmological timescales. With this requirement, scalar fields cannot be discarded as viable candidates for dark matter halo models in galaxies around central supermassive black holes (SMBH). One hypothesis for the formation of most SMBHs at high redshift is the gravitational collapse of supermassive stars (SMS) with masses of $\sim 10^{5} \mathrm{M}_{\odot}$. Therefore, a constraint for the existence of quasi-bound states of scalar fields is their survival to such dynamic events. To answer this question we present in this paper the results of a series of numerical relativity simulations of gravitationally collapsing, spherically symmetric stars surrounded by self-gravitating scalar fields. We use an ideal fluid equation of state with adiabatic index $\Gamma=4 / 3$ which is adequate to simulate radiation-dominated isentropic SMSs. Our results confirm the existence of oscillating, long-lived, self-gravitating scalar field configurations around non-rotating black holes after the collapse of the stars.

PACS numbers: 95.30.Lz, 95.30.Sf, 04.70.Bw, 04.25.dg
\end{abstract}

\section{INTRODUCTION}

The formation of galactic black holes is a fundamental issue in the study of galaxy formation. Most galaxies host black holes in their nuclei. Very luminous quasars at redshifts higher than 6 are believed to be powered by supermassive black holes (SMBH) with masses of $\sim$ $10^{9} \mathrm{M}_{\odot}$ [1, 2. However, it is still unknown how such SMBH can form and grow to reach the current values when the Universe was less than $10^{9}$ years old.

One possible scenario for the formation of SMBH in the center of galaxies is that they were formed through accretion and merging of the first stellar remnants [3, 4, Another scenario assumes that central black holes formed via direct collapse (see [5-7]). This route assumes that fragmentation, which depends on efficient cooling, is suppressed. Depending on the rate and efficiency of the inflowing mass, there may be different outcomes. A low rate of mass accumulation would favor the formation of isentropic SMSs, with masses $\geq 5 \times 10^{4} \mathrm{M}_{\odot}$, which then would evolve as equilibrium configurations dominated by radiation pressure before collapsing to form a SMBH seed [8, 9].

Scalar fields have been considered in cosmology to provide inflationary solutions in the early universe 10 and as an alternative explanation for dark energy [11. In addition, they have also been proposed as strong candidates for dark matter 12 [15. In these models one assumes that dark matter is composed by bosonic particles which may condensate into macroscopic objects forming boson stars or forming halos around black holes. The fact that most galaxies host a SMBH at their centers has raised many questions regarding the dynamics of the whole system.

The issue of whether scalar field halos are able to survive around black holes for cosmological timescales has been addressed in several occasions (see e.g. [16] and references therein). It has been shown in the test-field limit in the background spacetime of a Schwarzschild black hole, that scalar field configurations do remain around SMBHs for cosmological timescales, provided the mass of the scalar particle is of the order of $10^{-22}-10^{-24} \mathrm{eV}$. Further studies considered also the backreaction of spacetime [17-19, and confirmed the existence of such longlived states. These gravitationally trapped, scalar field configurations are states characterized by precise complex frequencies. It has been proved that these states are generic in the sense that they appear after some time in the evolution of the scalar field even when arbitrary initial data is used 20. This observation seems to be enough to guarantee (as we show below) that any choice of initial data will create a scalar field environment around the black hole.

The main objective of this work is to assess the role of black hole formation in the presence of scalar field configurations which resemble the quasi-bound states found in previous investigations where the black hole is assumed to exist from the start. To accomplish this goal we perform numerical relativity simulations which track the gravitational collapse of a polytropic star in the presence of a scalar field environment. The numerical techniques and the code used are those already described in [17. We shall refer to that work as Paper 1. In this work we also take into account the evolution of the relativistic hydro- 
dynamic equations. In order to simplify our model, we consider that the scalar field and the matter of the star are coupled only through gravity.

The paper is organized as follows: In Section II we present the basic equations and discuss the initial data used in our simulations. Section III briefly describes our numerical approach. In Section $\overline{I V}$ we discuss our findings and describe some properties of the solutions. Finally in Section $\mathrm{V}$ we sum up our concluding remarks. Throughout the paper Greek indices run over spacetime indices (0 to 3), while Latin indices run over space indices only (1 to 3 ). We use units in which $c=G=M_{\odot}=1$.

\section{BASIC EQUATIONS}

We investigate the dynamics of a self-gravitating scalar field configuration around a polytropic star collapsing to a black hole by solving numerically the coupled EinsteinKlein-Gordon system and the general relativistic hydrodynamics equations. The Einstein equations in covariant form read

$$
R_{\alpha \beta}-\frac{1}{2} g_{\alpha \beta} R=8 \pi T_{\alpha \beta},
$$

where $R_{\alpha \beta}$ is the Ricci tensor of the 4-dimensional spacetime, $g_{\alpha \beta}$ is the spacetime metric, $R$ is the Ricci scalar, and the matter content is given for our system by a combined stress-energy tensor

$$
T_{\alpha \beta}=T_{\alpha \beta}^{\mathrm{F}}+T_{\alpha \beta}^{\mathrm{SF}},
$$

where $T_{\alpha \beta}^{\mathrm{F}}$ is the stress-energy tensor of the (perfect) fluid defined by

$$
T_{\alpha \beta}^{\mathrm{F}}=\rho h u_{\alpha} u_{\beta}+p g_{\alpha \beta},
$$

and $T_{\alpha \beta}^{\mathrm{SF}}$ is the part corresponding to the scalar field, given by

$$
T_{\alpha \beta}^{\mathrm{SF}}=\partial_{\alpha} \Phi \partial_{\beta} \Phi-\frac{1}{2} g_{\alpha \beta}\left(\partial^{\sigma} \Phi \partial_{\sigma} \Phi+\mu^{2} \Phi^{2}\right) .
$$

In Eq. (3) $\rho$ is the rest-mass density, $p$ is the pressure, $u_{\alpha}$ is the fluid four velocity and $h=1+\varepsilon+p / \rho$ is the relativistic specific enthalpy, with $\varepsilon$ being the specific internal energy. We will assume that each type of matter obeys their own conservation law, in other words, that the coupling between them comes purely from gravity. The equations of motion for the scalar field are obtained from the Klein-Gordon equation

$$
\square \Phi-\mu^{2} \Phi=0,
$$

where the D'Alambertian operator is defined by $\square:=$ $(1 / \sqrt{-g}) \partial_{\alpha}\left(\sqrt{-g} g^{\alpha \beta} \partial_{\beta}\right)$. We follow the convention that $\Phi$ is dimensionless and $\mu$ has dimensions of (length) ${ }^{-1}$.

In the following we present the different systems of evolution (and constraint) equations we solve to carry out our study. While we mainly include this information to make the paper self-contained, we keep these sections as concise as possible, and refer the interested reader to our previous papers [17, 21, 22] for further details.

\section{A. Einstein's equations}

Under the assumption of spherical symmetry the $1+1$ line element may be written as

$$
d s^{2}=e^{4 \chi}\left(a(t, r) d t^{2}+r^{2} b(t, r) d \Omega^{2}\right),
$$

with $d \Omega^{2}=\sin ^{2} \theta d \varphi^{2}+d \theta^{2}$ being the solid angle element and $a(t, r)$ and $b(t, r)$ two non-vanishing metric functions. Moreover $\chi$ is related to the conformal factor $\psi$ as $\psi=$ $e^{\chi}=(\gamma / \hat{\gamma})^{1 / 12}$, with $\gamma$ and $\hat{\gamma}$ being the determinants of the physical and conformal 3 -metrics, respectively. They are conformally related by $\gamma_{i j}=e^{4 \chi} \hat{\gamma}_{i j}$.

In particular we adopt the BSSN formalism of Einstein's equations [23, 24, where the evolved fields are the conformally related 3 -dimensional metric, the conformal exponent $\chi$, the trace of the extrinsic curvature $K$, the independent component of the traceless part of the conformal extrinsic curvature, and the radial component of the conformal connection functions (we refer to [21, 25] for further details).

The matter fields appearing on the right-hand-side of the evolution equations for the gravitational fields (see [21, 25]), $\mathcal{E}, j_{i}, S_{i}$, and $S_{i j}$ include the contribution of both the fluid and the scalar field, i.e. $\mathcal{E}=\mathcal{E}^{\mathrm{F}}+\mathcal{E}^{\mathrm{SF}}$, etc. The matter source terms for the fluid given by the stress-energy tensor of Eq. (3) read

$$
\begin{aligned}
\mathcal{E}^{\mathrm{F}} & \equiv n^{\alpha} n^{\beta} T_{\alpha \beta}^{\mathrm{F}}, \\
j_{r}^{\mathrm{F}} & \equiv-\gamma_{r}^{\alpha} n^{\beta} T_{\alpha \beta}^{\mathrm{F}}, \\
S_{a}^{\mathrm{F}} & \equiv\left(T^{\mathrm{F}}\right)_{r}^{r}, \\
S_{b}^{\mathrm{F}} & \equiv\left(T^{\mathrm{F}}\right)_{\theta}^{\theta} .
\end{aligned}
$$

The Hamiltonian and momentum constraints are given by the following two equations that we compute to monitor the accuracy of the numerical evolutions:

$$
\begin{aligned}
\mathcal{H} & \equiv R-\left(A_{a}^{2}+2 A_{b}^{2}\right)+\frac{2}{3} K^{2}-16 \pi \mathcal{E}=0, \\
\mathcal{M}_{r} & \equiv \partial_{r} A_{a}-\frac{2}{3} \partial_{r} K+6 A_{a} \partial_{r} \chi \\
& +\left(A_{a}-A_{b}\right)\left(\frac{2}{r}+\frac{\partial_{r} b}{b}\right)-8 \pi j_{r}=0
\end{aligned}
$$

where $A_{a}$ and $A_{b}$ are the contraction of the traceless part of the conformal extrinsic curvature, $A_{a} \equiv \hat{A}_{r}^{r}, A_{b} \equiv$ $\hat{A}_{\theta}^{\theta}$. In addition to the evolution fields, there are two more variables left undetermined, the lapse function $\alpha$, and the shift vector $\beta^{i}$. Our code can handle arbitrary gauge conditions and for the simulations reported in this paper we use the same conditions of Paper 1, namely the socalled "non-advective 1+log" condition [26] for the lapse, and a variation of the "Gamma-driver" condition for the shift vector [25, 27]. 


\section{B. Klein-Gordon equation}

To solve the Klein-Gordon equation we introduce two first-order variables defined as:

$$
\begin{aligned}
& \Pi:=n^{\alpha} \partial_{\alpha} \Phi=\frac{1}{\alpha}\left(\partial_{t} \Phi-\beta^{r} \partial_{r} \Phi\right) \\
& \Psi:=\partial_{r} \Phi
\end{aligned}
$$

Therefore, using Eq. (5) we obtain the following system of first-order equations:

$$
\begin{aligned}
\partial_{t} \Phi & =\beta^{r} \partial_{r} \Phi+\alpha \Pi, \\
\partial_{t} \Psi & =\beta^{r} \partial_{r} \Psi+\Psi \partial_{r} \beta^{r}+\partial_{r}(\alpha \Pi), \\
\partial_{t} \Pi & =\beta^{r} \partial_{r} \Pi+\frac{\alpha}{a e^{4 \chi}}\left[\partial_{r} \Psi\right. \\
& \left.+\Psi\left(\frac{2}{r}-\frac{\partial_{r} a}{2 a}+\frac{\partial r b}{b}+2 \partial_{r} \chi\right)\right] \\
& +\frac{\Psi}{a e^{4 \chi}} \partial_{r} \alpha+\alpha K \Pi-\alpha \mu^{2} \Phi .
\end{aligned}
$$

The matter source terms for the scalar field read

$$
\begin{aligned}
\mathcal{E}^{\mathrm{SF}} & \equiv n^{\alpha} n^{\beta} T_{\alpha \beta}^{\mathrm{SF}}=\frac{1}{2}\left(\Pi^{2}+\frac{\Psi^{2}}{a e^{4 \chi}}\right)+\frac{1}{2} \mu^{2} \Phi^{2}, \\
j_{r}^{\mathrm{SF}} & \equiv-\gamma_{r}^{\alpha} n^{\beta} T_{\alpha \beta}^{\mathrm{SF}}=-g_{r r} \Pi \Psi \\
S_{a}^{\mathrm{SF}} & \equiv\left(T^{\mathrm{SF}}\right)_{r}^{r}=\frac{1}{2}\left(\Pi^{2}+\frac{\Psi^{2}}{a e^{4 \chi}}\right)-\frac{1}{2} \mu^{2} \Phi^{2}, \\
S_{b}^{\mathrm{SF}} & \equiv\left(T^{\mathrm{SF}}\right)_{\theta}^{\theta}=\frac{1}{2}\left(\Pi^{2}-\frac{\Psi^{2}}{a e^{4 \chi}}\right)-\frac{1}{2} \mu^{2} \Phi^{2} .
\end{aligned}
$$

\section{Hydrodynamics equations}

The general relativistic hydrodynamics equations, expressed through the conservation laws of the stressenergy tensor $T^{\mu \nu}$ and the continuity equation, are

$$
\nabla_{\alpha} T^{\alpha \beta}=0, \quad \nabla_{\alpha}\left(\rho u^{\alpha}\right)=0 .
$$

Following [28, we write the equations of general relativistic hydrodynamics as a first-order, flux-conservative system. We define the fluid 3-velocity as seen by a normal (Eulerian) observer as

$$
v^{r} \equiv \frac{u^{r}}{\alpha u^{t}}+\frac{\beta^{r}}{\alpha}
$$

and the Lorentz factor between the fluid and the normal observer as $W \equiv \alpha u^{t}$. We also define the relativistic densities of mass, momentum, and energy for the normal observer as

$$
\begin{aligned}
D & =\rho W, \\
S_{r} & =\rho h W^{2} v_{r}, \\
\tau & =\rho h W^{2}-p-D .
\end{aligned}
$$

We then assemble these variables into a vector $\mathbf{U}$ of conserved fluid variables

$$
\mathbf{U}=\sqrt{\gamma}\left(D, S_{r}, \tau\right)
$$

By defining the corresponding vector of fluxes $\mathbf{F}^{r}$ and the vector of source terms $\mathbf{S}$ we can cast Eqs. (22) in flux-conservative form

$$
\partial_{t} \mathbf{U}+\partial_{r} \mathbf{F}^{r}=\mathbf{S}
$$

We refer to 21] where the explicit form of vectors $\mathbf{F}^{r}$ and $\mathbf{S}$ is given. To close the system of hydrodynamics equations, we choose a Gamma-law equation of state (EOS)

$$
P=(\Gamma-1) \rho \epsilon,
$$

where $\Gamma$ is the adiabatic index.

\section{Scalar field initial data}

As in Paper I our choice of initial data for the scalar field is a Gaussian distribution of the form

$$
\Phi=A_{0} e^{-\left(r-r_{0}\right)^{2} / \lambda^{2}}
$$

where $A_{0}$ is the initial amplitude of the pulse, $r_{0}$ is the center of the Gaussian, and $\lambda$ is its width. The auxiliary first-order quantities are initialized as follows

$$
\begin{aligned}
& \Pi(t=0, r)=0, \\
& \Psi(t=0, r)=-2 \frac{\left(r-r_{0}\right)}{\lambda^{2}} A_{0} e^{-\left(r-r_{0}\right)^{2} / \lambda^{2}} .
\end{aligned}
$$

Likewise, we choose a conformally flat metric with $a=b=1$ together with a time symmetry condition $K_{i j}=0$. With these choices and $\Pi(t=0, r)=0$ the momentum constraint is satisfied trivially and the Hamiltonian constraint, Eq. (11), yields the following equation for the conformal factor $\psi=e^{\chi}$,

$$
\partial_{r r} \psi+\frac{2}{r} \partial_{r} \psi+2 \pi \psi^{5} \mathcal{E}=0
$$

In order to solve this equation for our collapsing stars, we assume that the conformal factor can be written as

$$
\psi=\psi^{\mathrm{TOV}}+u(r)
$$

where $\psi^{\mathrm{TOV}}$ is the part of the conformal factor associated with the stellar Tolman-Oppenheimer-Volkoff (TOV) model. By substituting this ansatz in the Hamiltonian constraint we obtain

$\partial_{r r} u(r)+\frac{2}{r} \partial_{r} u(r)+2 \pi \psi^{5} \mathcal{E}^{\mathrm{SF}}+2 \pi\left(\psi^{5}-\left(\psi^{\mathrm{TOV}}\right)^{5}\right) \mathcal{E}^{\mathrm{TOV}}=0$

Given a distribution of the scalar field density $\mathcal{E}^{\mathrm{SF}}$, we solve this ordinary differential equation for $u$ using a standard fourth-order Runge-Kutta integrator, assuming that $u \rightarrow 0$ as $r \rightarrow \infty$ and regularity at the origin. 
TABLE I: Stellar TOV models: $N=3(\Gamma=4 / 3)$ polytropes with $\kappa=0.48773$. From left to right the columns indicate the model number, the central rest-mass density, $\rho_{c}$, the central energy density, $e_{c}$, the radius of the star, $R$, the gravitational mass of the star, $M$, and the compactness, $M / R$.

\begin{tabular}{cccccc}
\hline \hline Model & $\rho_{c}$ & $e_{c}$ & $R$ & $M$ & $M / R$ \\
\hline 1 & $3.11 \mathrm{e}-6$ & $3.17 \mathrm{e}-6$ & 185.8 & 1.43 & 0.008 \\
2 & $3.11 \mathrm{e}-5$ & $3.25 \mathrm{e}-5$ & 86.3 & 1.31 & 0.015 \\
3 & $3.11 \mathrm{e}-4$ & $3.41 \mathrm{e}-4$ & 39.9 & 1.01 & 0.025 \\
4 & $3.11 \mathrm{e}-3$ & $3.77 \mathrm{e}-3$ & 19.2 & 0.80 & 0.042 \\
\hline \hline
\end{tabular}

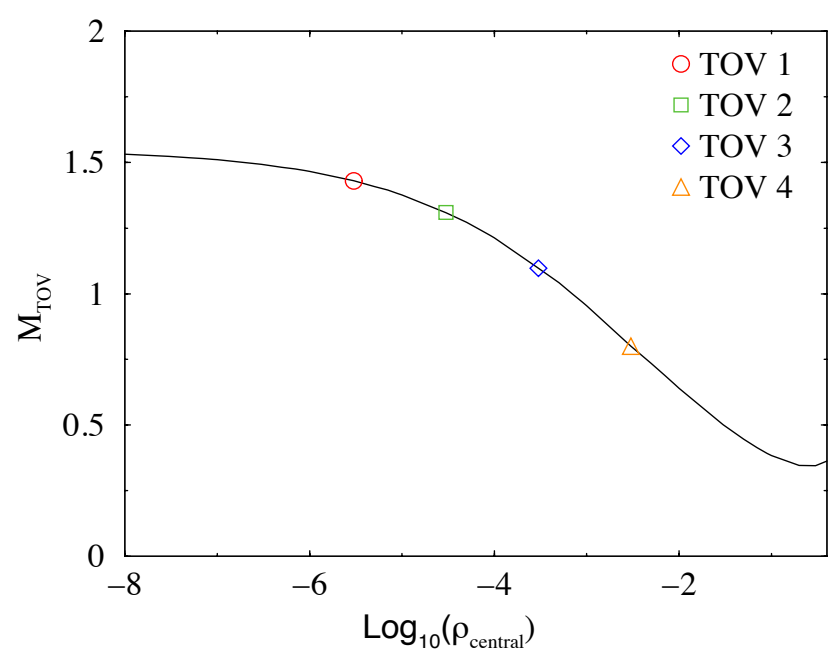

FIG. 1: Mass of the polytropic TOV stars as function of their initial central rest-mass density. All models are in the unstable branch of the diagram.

\section{NUMERICS}

The time update of the different systems of evolution equations we have to solve in our code (Einstein, KleinGordon, and Euler) is done using the same type of techniques we have extensively used in our previous work (see in particular [17, 21]). We address the interested reader to those references for full details on the particular numerical techniques implemented in the code. Here, we simply mention that the evolution equations are integrated using the second-order PIRK method developed by [29, 30. This method allows to handle the singular terms that appear in the evolution equations due to our choice of curvilinear coordinates. The derivatives in the spacetime evolution are computed using a fourthorder centered finite difference approximation on a uniform grid except for advection terms for which we adopt a fourth-order upwind scheme. We also use fourth-order Kreiss-Oliger dissipation to avoid high frequency noise appearing near the outer boundary. The difference with our previous work [17 is that we are also evolving the hydrodynamics conserved quantities explicitly. Corre- spondingly, the equations of hydrodynamics are solved using the HLLE approximate Riemann solver in tandem with the second-order MC reconstruction scheme 21.

\section{RESULTS}

\section{A. Stellar models and code convergence}

We construct spherically-symmetric stellar models by solving the Tolman-Oppenheimer-Volkoff (TOV) equations for a polytropic EOS,

$$
p=\kappa \rho^{1+1 / N},
$$

where $\kappa$ is the polytropic constant and $N$ the polytropic index. These data are evolved in our code with the Gamma-law EOS, Eq. (29), with $\Gamma=1+1 / N$. We adopt $N=3(\Gamma=4 / 3)$, which is the appropriate value for a SMS. Moreover, we select the value of $\kappa=0.48773$ in our code units. The polytropic constant is set to this value to keep the mass of the star close to one, namely in the range $M=\{0.8,1.43\}$ for our four different TOV models. These models are gravitationally unstable spherical relativistic stars that collapse to black holes in dynamical timescales by artificially decreasing the pressure by a small amount (typically 5\%) at $t=0$. The main characteristics of our sample of stellar models are reported in Table I. Their corresponding location in the mass vs central density diagram is displayed in Fig. 1. The models are constructed using the RNS code [31].

The collapsing polytropic stars are surrounded by a scalar field cloud whose functional form is given by Eq. (30). We investigate the behaviour of the scalar field in a highly dynamical evolution in which the stars undergo gravitational collapse. The end result of the collapse is the formation of a Schwarzschild black hole whose mass is the sum of the mass of the corresponding original star and a portion of the scalar field mass, the part that is accreted. The aim of this investigation is to find out whether in such a dynamical scenario, part of the scalar field can still survive in the form of long-lived quasi-bound states.

To carry out our study we set up and evolve 80 different initial scalar field models. These models are characterized by different values of the initial energy of the scalar field, namely $E_{0}=\left\{3.2 \times 10^{-5}, 0.50,2.85,4.3\right\}$ and different values of the scalar field mass $\mu=$ $\{0.05,0.08,0.1,0.15,0.2\}$. The choice of the values for the energy is made to span all regimes of interest, from the test-field regime to that for which the self-gravity of the scalar field becomes important. For all models, the distribution of the scalar field pulse is placed at the same initial location $r_{0}=100$ and they all share the same initial width $\lambda=50$.

We have used a logarithmic radial grid in our simulations setting to $\Delta r=0.1$ the minimum radial resolution at the origin. This is different from the grid we used in 


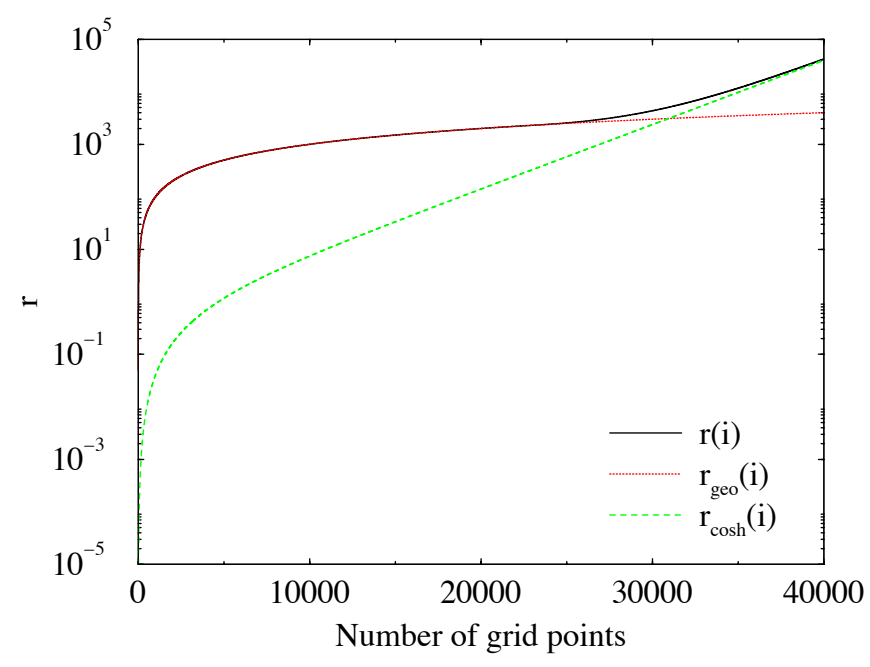

FIG. 2: Extent of the radial grid as a function of the number of grid points. The black solid line represents the radial coordinate profile of the composite grid, the red dotted line the geometric progression patch and the green dashed line the hyperbolic cosine patch.

Paper 1. The reason behind this choice is to reduce the number of radial points without loosing accuracy in the inner region and placing the outer boundary sufficiently far away to avoid unwanted reflections from the scalar field. Our grid is composed of two patches, a geometrical progression in the interior and a hyperbolic cosine outside. Using the inner grid alone requires too many grid points to place the outer boundary sufficiently far from the origin, while the hyperbolic cosine patch produces very small grid spacings in the inner region of the grid, leading to prohibitively small timesteps due to the CFL condition. The two patches are defined at each radial point as

$$
\begin{aligned}
r_{\text {geo }}(i) & =i \Delta r g_{\text {prog }}^{i}-r_{\text {min }} \\
r_{\text {cosh }}(i) & =\cosh \left[\mathrm { i } \left(-\log \left(\left(1+\mathrm{r}_{\max }\right)\right.\right.\right. \\
& \left.\left.\left.+\sqrt{2 r_{\max }+r_{\max }^{2}}\right) /\left(N_{r}\right)\right)\right]-1,
\end{aligned}
$$

where $\Delta r$ is the minimum resolution, $r_{\min }$ and $r_{\max }$ the inner and outer boundary respectively, and $N_{r}$ the total number of radial points. The outer boundary of the computational domain is placed at $r_{\max }=4 \times 10^{4}$, far enough as to not affect the dynamics in the inner region during the entire simulation. For the matching of the two patches, we compute the grid spacings $\Delta r_{i}$ for the two patches $r_{\text {geo }}(i)$ and $r_{\text {cosh }}(i)$ at each grid point and when the difference between the grid spacings is at a minimum, we change from the interior grid to the hyperbolic cosine grid from the corresponding radial point onwards. In Fig. 2 we plot the two patches and the resulting grid as a function of the number of grid points. The time step is given by $\Delta t=0.5 \Delta r$ in order to obtain long-term stable simulations. The final time of the numerical evolutions

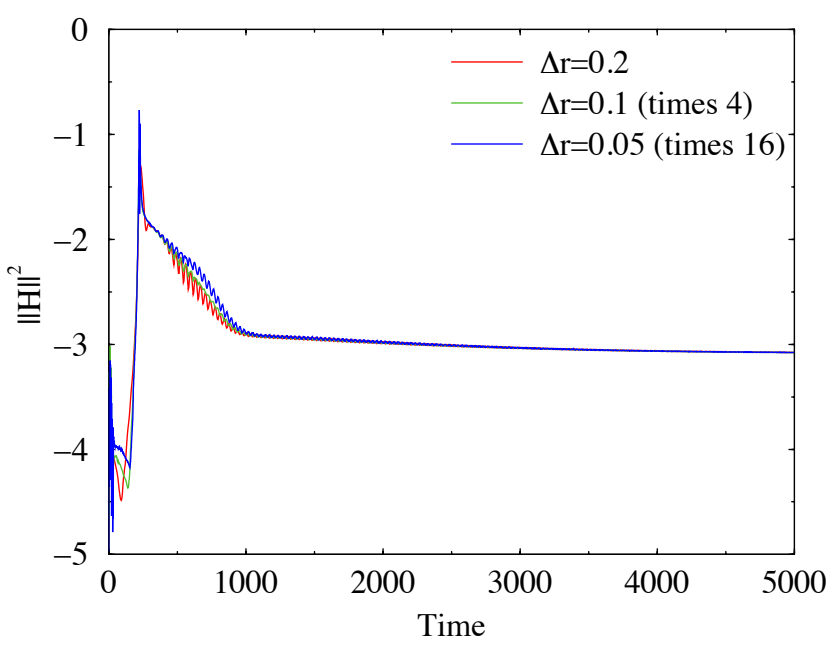

FIG. 3: Time evolution of the L2 norm of the Hamiltonian constraint for an initial scalar field pulse with $E_{0}=5 \times 10^{-3}$ and mass $M \mu=0.1$ for the TOV model 4. The plot shows results for three different resolutions, rescaled by the factors corresponding to second-order convergence.

is $3.5 \times 10^{4}$.

While the convergence of the code was already satisfactorily tested in Paper 1, we nevertheless need to assess it again here since we now deal with an augmented system of equations than in Paper 1 due to the presence of the hydrodynamical terms. In order to test the convergence of the code we performed three simulations with different resolutions $\Delta r=\{0.2,0.1,0.05\}$. In Fig. 3 we plot the rescaled evolution of the L2 norm of the Hamiltonian constraint for a particular choice of the initial energy, $E_{0}=4.3$, scalar field mass, $\mu=0.1$, and for the TOV model 4 , obtaining the expected second-order convergence of our PIRK time-evolution scheme. We note that the same result is achieved irrespective of the combination of parameters considered and of the TOV model.

Comparing simulations with different resolutions, we estimate that the error in the final black hole mass is $\sim 2 \%$ while the error estimates for the scalar field energy and ADM mass at the end of the simulations are $\sim 2-3 \%$. Those errors decrease as $(\Delta r)^{2}$ with increasing resolution. Taking into account that our simulations are performed up to a (significantly long) final time of $3.5 \times 10^{4}$, the error bars for our main quantifiable quantity, the frequencies of oscillation of the scalar field (see next section), are $\sim 3 \times 10^{-5}$.

\section{B. Non-linear quasi-bound states}

We solve the Einstein-Klein-Gordon-Euler system using the initial data given by Eqs. (30)- $\sqrt{32}$ and let the scalar field evolve in the rapidly changing gravitational field of a collapsing polytropic star. As in Paper 1 we an- 
TABLE II: Initial parameters and most relevant quantities for the scalar field and collapsing TOV model $1(M=1.43)$. From left to right the columns report: the name of the model, the initial amplitude of the pulse, $A_{0}$, the scalar field mass, $\mu$, the real part of the angular frequency $\omega$ for the fundamental mode of oscillation and the first overtone, the time of the collapse of the TOV star and the formation of the black hole, $t_{\mathrm{col}}$, the final mass of the apparent horizon of the black hole, $M_{\mathrm{AH}}$, the initial $\mathrm{ADM}$ mass of the system, $M_{\mathrm{ADM}}$, and the initial and final energy of the scalar field, $E_{0}$ and $E_{\text {final }}$ respectively. The initial Gaussian pulse is located at $r_{0}=100$ with half-width $\lambda=50$.

\begin{tabular}{|c|c|c|c|c|c|c|c|c|c|}
\hline \multirow[t]{2}{*}{ Model } & \multirow[t]{2}{*}{$A_{0}$} & \multirow[t]{2}{*}{$\mu$} & \multicolumn{2}{|c|}{$\omega$} & \multirow[t]{2}{*}{$t_{\mathrm{col}}$} & \multirow[t]{2}{*}{$M_{\mathrm{AH}}$} & \multirow[t]{2}{*}{$M_{\mathrm{ADM}}$} & \multirow[t]{2}{*}{$E_{0}$} & \multirow[t]{2}{*}{$E_{\text {final }}$} \\
\hline & & & 1 & 2 & & & & & \\
\hline 1_1a & $5.00 \mathrm{E}-5$ & 0.05 & 0.04990 & $\ldots$ & 2494 & 1.40 & 1.43 & $3.2 \mathrm{E}-5$ & $3.1 \mathrm{E}-5$ \\
\hline $1 \_1 b$ & $3.30 \mathrm{E}-5$ & 0.08 & 0.07935 & 0.07988 & 2494 & 1.40 & 1.43 & $3.2 \mathrm{E}-5$ & $2.0 \mathrm{E}-5$ \\
\hline $1 \_1 \mathrm{c}$ & $2.65 \mathrm{E}-5$ & 0.10 & 0.09874 & 0.09964 & 2494 & 1.40 & 1.43 & $3.2 \mathrm{E}-5$ & $1.2 \mathrm{E}-5$ \\
\hline 1_1d & $1.80 \mathrm{E}-5$ & 0.15 & 0.14901 & 0.14954 & 2494 & 1.40 & 1.43 & $3.2 \mathrm{E}-5$ & $1.2 \mathrm{E}-5$ \\
\hline $1 \_1 \mathrm{e}$ & $1.35 \mathrm{E}-5$ & 0.20 & 0.19783 & 0.19927 & 2494 & 1.40 & 1.43 & $3.2 \mathrm{E}-5$ & $5.0 \mathrm{E}-6$ \\
\hline 1_2a & $6.30 \mathrm{E}-3$ & 0.05 & 0.05009 & $\ldots$ & 1706 & 1.40 & 1.92 & 0.50 & 0.49 \\
\hline 1_2b & $4.12 \mathrm{E}-3$ & 0.08 & 0.07953 & 0.08007 & 1582 & 1.67 & 1.92 & 0.50 & 0.22 \\
\hline $1 \_2 \mathrm{c}$ & $3.33 \mathrm{E}-3$ & 0.10 & 0.09859 & 0.09999 & 1508 & 1.76 & 1.92 & 0.50 & 0.15 \\
\hline $1 \_2 \mathrm{~d}$ & $2.25 \mathrm{E}-3$ & 0.15 & 0.15008 & $\ldots$ & 1376 & 1.77 & 1.92 & 0.50 & 0.14 \\
\hline $1 \_2 \mathrm{e}$ & $1.70 \mathrm{E}-3$ & 0.20 & 0.20017 & $\ldots$ & 1306 & 1.90 & 1.92 & 0.50 & 0.02 \\
\hline 1_3a & $1.50 \mathrm{E}-2$ & 0.05 & 0.05099 & $\ldots$ & 774 & 1.49 & 4.20 & 2.85 & 2.71 \\
\hline 1_3b & $9.82 \mathrm{E}-3$ & 0.08 & 0.07909 & 0.08115 & 748 & 3.38 & 4.19 & 2.84 & 0.81 \\
\hline $1 \_3 \mathrm{c}$ & $7.95 \mathrm{E}-3$ & 0.10 & 0.10197 & $\ldots$ & 738 & 3.47 & 4.19 & 2.84 & 0.70 \\
\hline 1_3d & $5.36 \mathrm{E}-3$ & 0.15 & 0.15314 & $\ldots$ & 728 & 4.10 & 4.19 & 2.84 & 0.07 \\
\hline 1_3e & $4.04 \mathrm{E}-3$ & 0.20 & 0.20412 & $\ldots$ & 730 & 4.13 & 4.20 & 2.84 & 0.02 \\
\hline 1_4a & $1.85 \mathrm{E}-2$ & 0.05 & 0.05153 & $\ldots$ & 606 & 1.83 & 5.72 & 4.31 & 3.81 \\
\hline 1_4b & $1.22 \mathrm{E}-2$ & 0.08 & 0.08276 & $\ldots$ & 608 & 4.49 & 5.77 & 4.36 & 1.18 \\
\hline $1 \_4 \mathrm{c}$ & $9.85 \mathrm{E}-3$ & 0.10 & 0.10341 & $\ldots$ & 612 & 5.06 & 5.75 & 4.34 & 0.54 \\
\hline $1 \_4 d$ & $6.65 \mathrm{E}-3$ & 0.15 & 0.15512 & $\ldots$ & 626 & 5.53 & 5.76 & 4.35 & 0.07 \\
\hline $1 \_4 \mathrm{e}$ & $5.00 \mathrm{E}-3$ & 0.20 & 0.20665 & $\ldots$ & 642 & 5.60 & 5.73 & 4.33 & 0.007 \\
\hline
\end{tabular}

TABLE III: Same as Table మut for TOV model 2.

\begin{tabular}{|c|c|c|c|c|c|c|c|c|c|}
\hline \multirow[t]{2}{*}{ Model } & \multirow[t]{2}{*}{$A_{0}$} & \multirow[t]{2}{*}{$\mu$} & \multicolumn{2}{|c|}{$\omega$} & \multirow[t]{2}{*}{$t_{\mathrm{col}}$} & \multirow[t]{2}{*}{$M_{\mathrm{AH}}$} & \multirow[t]{2}{*}{$M_{\mathrm{ADM}}$} & \multirow[t]{2}{*}{$E_{0}$} & \multirow[t]{2}{*}{$E_{\text {final }}$} \\
\hline & & & 1 & 2 & & & & & \\
\hline $2 \_1 \mathrm{a}$ & $5.00 \mathrm{E}-5$ & 0.05 & 0.04991 & $\ldots$ & 750 & 1.28 & 1.31 & $3.2 \mathrm{E}-5$ & $3.1 \mathrm{E}-5$ \\
\hline $2 \_1 \mathrm{~b}$ & $3.30 \mathrm{E}-5$ & 0.08 & 0.07953 & $\ldots$ & 750 & 1.28 & 1.31 & $3.2 \mathrm{E}-5$ & $2.1 \mathrm{E}-5$ \\
\hline 2_1c & $2.65 \mathrm{E}-5$ & 0.10 & 0.09909 & 0.09981 & 750 & 1.28 & 1.31 & $3.2 \mathrm{E}-5$ & $1.0 \mathrm{E}-5$ \\
\hline $2 \_1 d$ & $1.80 \mathrm{E}-5$ & 0.15 & 0.14650 & 0.14918 & 750 & 1.28 & 1.31 & $3.2 \mathrm{E}-5$ & $9.9 \mathrm{E}-6$ \\
\hline 2_1e & $1.35 \mathrm{E}-5$ & 0.20 & 0.19802 & 0.19927 & 750 & 1.28 & 1.31 & $3.2 \mathrm{E}-5$ & $2.7 \mathrm{E}-6$ \\
\hline $2 \_2 \mathrm{a}$ & $6.30 \mathrm{E}-3$ & 0.05 & 0.05009 & $\ldots$ & 640 & 1.28 & 1.80 & 0.50 & 0.49 \\
\hline $2 \_2 \mathrm{~b}$ & $4.12 \mathrm{E}-3$ & 0.08 & 0.07971 & 0.08025 & 650 & 1.54 & 1.80 & 0.50 & 0.23 \\
\hline $2 \_2 \mathrm{c}$ & $3.33 \mathrm{E}-3$ & 0.10 & 0.09859 & 0.10008 & 654 & 1.64 & 1.80 & 0.50 & 0.16 \\
\hline 2_2d & $2.25 \mathrm{E}-3$ & 0.15 & 0.14936 & 0.15009 & 660 & 1.67 & 1.80 & 0.50 & 0.10 \\
\hline $2 \_2 \mathrm{e}$ & $1.70 \mathrm{E}-3$ & 0.20 & 0.20017 & $\ldots$ & 666 & 1.76 & 1.80 & 0.50 & 0.03 \\
\hline 2_3a & $1.50 \mathrm{E}-2$ & 0.05 & 0.05117 & $\ldots$ & 438 & 1.36 & 4.07 & 2.84 & 2.71 \\
\hline $2 \_3 b$ & $9.82 \mathrm{E}-3$ & 0.08 & 0.07918 & 0.08170 & 472 & 3.20 & 4.07 & 2.83 & 0.90 \\
\hline $2 \_3 c$ & $7.95 \mathrm{E}-3$ & 0.10 & 0.10197 & $\ldots$ & 488 & 3.39 & 4.07 & 2.83 & 0.65 \\
\hline $2 \_3 \mathrm{~d}$ & $5.36 \mathrm{E}-3$ & 0.15 & 0.15314 & $\ldots$ & 516 & 3.98 & 4.07 & 2.83 & 0.09 \\
\hline $2 \_3 \mathrm{e}$ & $4.04 \mathrm{E}-3$ & 0.20 & 0.20412 & $\ldots$ & 536 & 4.01 & 4.07 & 2.83 & 0.02 \\
\hline 2_4a & $1.85 \mathrm{E}-2$ & 0.05 & 0.05170 & $\ldots$ & 386 & 1.58 & 5.60 & 4.30 & 3.92 \\
\hline $2 \_4 \mathrm{~b}$ & $1.22 \mathrm{E}-2$ & 0.08 & 0.08276 & $\ldots$ & 426 & 4.32 & 5.65 & 4.35 & 1.18 \\
\hline $2 \_4 \mathrm{c}$ & $9.85 \mathrm{E}-3$ & 0.10 & 0.10341 & $\ldots$ & 444 & 5.05 & 5.63 & 4.33 & 0.44 \\
\hline $2 \_4 \mathrm{~d}$ & $6.65 \mathrm{E}-3$ & 0.15 & 0.15512 & $\ldots$ & 478 & 5.40 & 5.63 & 4.33 & 0.08 \\
\hline $2 \_4 \mathrm{e}$ & $5.00 \mathrm{E}-3$ & 0.20 & 0.20678 & $\ldots$ & 500 & 5.48 & 5.62 & 4.32 & 0.008 \\
\hline
\end{tabular}


TABLE IV: Same as Table II but for TOV model 3.

\begin{tabular}{|c|c|c|c|c|c|c|c|c|c|}
\hline \multirow[t]{2}{*}{ Model } & \multirow[t]{2}{*}{$A_{0}$} & \multirow[t]{2}{*}{$\mu$} & \multicolumn{2}{|c|}{$\omega$} & \multirow[t]{2}{*}{$t_{\mathrm{col}}$} & \multirow[t]{2}{*}{$M_{\mathrm{AH}}$} & \multirow[t]{2}{*}{$M_{\mathrm{ADM}}$} & \multirow[t]{2}{*}{$E_{0}$} & \multirow[t]{2}{*}{$E_{\text {final }}$} \\
\hline & & & 1 & 2 & & & & & \\
\hline 3_1a & $5.00 \mathrm{E}-5$ & 0.05 & 0.04991 & $\ldots$ & 228 & 1.09 & 1.10 & $3.2 \mathrm{E}-5$ & $3.1 \mathrm{E}-5$ \\
\hline 3_1b & $3.30 \mathrm{E}-5$ & 0.08 & 0.07971 & $\ldots$ & 228 & 1.09 & 1.10 & $3.2 \mathrm{E}-5$ & $2.6 \mathrm{E}-5$ \\
\hline 3_1c & $2.65 \mathrm{E}-5$ & 0.10 & 0.09928 & 0.09982 & 228 & 1.09 & 1.10 & $3.2 \mathrm{E}-5$ & $1.4 \mathrm{E}-5$ \\
\hline 3_1d & $1.80 \mathrm{E}-5$ & 0.15 & 0.14758 & 0.14936 & 228 & 1.09 & 1.10 & $3.2 \mathrm{E}-5$ & $1.1 \mathrm{E}-5$ \\
\hline 3_1e & $1.35 \mathrm{E}-5$ & 0.20 & 0.19855 & 0.19945 & 228 & 1.09 & 1.10 & $3.2 \mathrm{E}-5$ & $3.1 \mathrm{E}-6$ \\
\hline $3 \_2 \mathrm{a}$ & $6.30 \mathrm{E}-3$ & 0.05 & 0.05009 & $\ldots$ & 226 & 1.10 & 1.60 & 0.50 & 0.49 \\
\hline 3_2b & $4.12 \mathrm{E}-3$ & 0.08 & 0.07989 & $\ldots$ & 228 & 1.22 & 1.60 & 0.50 & 0.36 \\
\hline $3 \_2 \mathrm{c}$ & $3.33 \mathrm{E}-3$ & 0.10 & 0.09928 & 0.10018 & 228 & 1.40 & 1.60 & 0.50 & 0.16 \\
\hline $3 \_2 \mathrm{~d}$ & $2.25 \mathrm{E}-3$ & 0.15 & 0.14953 & 0.15026 & 228 & 1.50 & 1.60 & 0.50 & 0.09 \\
\hline $3 \_2 \mathrm{e}$ & $1.70 \mathrm{E}-3$ & 0.20 & 0.19827 & 0.20017 & 228 & 1.53 & 1.60 & 0.50 & 0.04 \\
\hline 3_3a & $1.50 \mathrm{E}-2$ & 0.05 & 0.05117 & $\ldots$ & 220 & 1.13 & 3.91 & 2.82 & 2.71 \\
\hline 3_3b & $9.82 \mathrm{E}-3$ & 0.08 & 0.07953 & 0.08133 & 226 & 2.94 & 3.91 & 2.82 & 0.91 \\
\hline $3 \_3 \mathrm{c}$ & $7.95 \mathrm{E}-3$ & 0.10 & 0.10197 & $\ldots$ & 226 & 3.22 & 3.91 & 2.82 & 0.61 \\
\hline 3_3d & $5.36 \mathrm{E}-3$ & 0.15 & 0.15314 & $\ldots$ & 228 & 3.72 & 3.90 & 2.81 & 0.11 \\
\hline 3_3e & $4.04 \mathrm{E}-3$ & 0.20 & 0.20412 & $\ldots$ & 228 & 3.82 & 3.91 & 2.82 & 0.03 \\
\hline 3_4a & $1.85 \mathrm{E}-2$ & 0.05 & 0.05170 & $\ldots$ & 218 & 1.23 & 5.35 & 4.28 & 4.02 \\
\hline 3_4b & $1.22 \mathrm{E}-2$ & 0.08 & 0.08276 & $\ldots$ & 226 & 4.15 & 5.39 & 4.32 & 1.15 \\
\hline $3 \_4 \mathrm{c}$ & $9.85 \mathrm{E}-3$ & 0.10 & 0.10341 & $\ldots$ & 226 & 4.82 & 5.35 & 4.30 & 0.43 \\
\hline 3_4d & $6.65 \mathrm{E}-3$ & 0.15 & 0.15512 & $\ldots$ & 228 & 5.17 & 5.38 & 4.31 & 0.08 \\
\hline $3 \_4 \mathrm{e}$ & $5.00 \mathrm{E}-3$ & 0.20 & 0.20682 & $\ldots$ & 228 & 5.29 & 5.37 & 4.29 & 0.01 \\
\hline
\end{tabular}

TABLE V: Same as Table II but for TOV model 4.

\begin{tabular}{|c|c|c|c|c|c|c|c|c|c|}
\hline \multirow[t]{2}{*}{ Model } & \multirow[t]{2}{*}{$A_{0}$} & \multirow[t]{2}{*}{$\mu$} & \multicolumn{2}{|c|}{$\omega$} & \multirow[t]{2}{*}{$t_{\mathrm{col}}$} & \multirow[t]{2}{*}{$M_{\mathrm{AH}}$} & \multirow[t]{2}{*}{$M_{\mathrm{ADM}}$} & \multirow[t]{2}{*}{$E_{0}$} & \multirow[t]{2}{*}{$E_{\text {final }}$} \\
\hline & & & 1 & 2 & & & & & \\
\hline 4_1a & $5.00 \mathrm{E}-5$ & 0.05 & 0.04991 & $\ldots$ & 72 & 0.75 & 0.80 & $3.2 \mathrm{E}-5$ & $3.1 \mathrm{E}-5$ \\
\hline 4_1b & $3.30 \mathrm{E}-5$ & 0.08 & 0.07989 & $\ldots$ & 72 & 0.75 & 0.80 & $3.2 \mathrm{E}-5$ & $3.1 \mathrm{E}-5$ \\
\hline 4_1c & $2.25 \mathrm{E}-5$ & 0.10 & 0.09963 & $\ldots$ & 72 & 0.75 & 0.80 & $2.3 \mathrm{E}-5$ & $1.9 \mathrm{E}-5$ \\
\hline 4_1d & $1.80 \mathrm{E}-5$ & 0.15 & 0.14882 & 0.14973 & 72 & 0.75 & 0.80 & $3.2 \mathrm{E}-5$ & $1.4 \mathrm{E}-5$ \\
\hline 4_1e & $1.35 \mathrm{E}-5$ & 0.20 & 0.19927 & 0.19963 & 72 & 0.75 & 0.80 & $3.2 \mathrm{E}-5$ & $7.5 \mathrm{E}-5$ \\
\hline $4 \_2 \mathrm{a}$ & $6.30 \mathrm{E}-3$ & 0.05 & 0.05009 & $\ldots$ & 72 & 0.75 & 1.29 & 0.50 & 0.49 \\
\hline $4 \_2 \mathrm{~b}$ & $4.12 \mathrm{E}-3$ & 0.08 & 0.08007 & $\ldots$ & 72 & 0.77 & 1.29 & 0.50 & 0.47 \\
\hline $4 \_2 \mathrm{c}$ & $3.33 \mathrm{E}-3$ & 0.10 & 0.09982 & 0.10035 & 72 & 0.89 & 1.28 & 0.49 & 0.35 \\
\hline $4 \_2 d$ & $2.25 \mathrm{E}-3$ & 0.15 & 0.14829 & 0.15008 & 72 & 1.09 & 1.29 & 0.50 & 0.19 \\
\hline $4 \_2 \mathrm{e}$ & $1.70 \mathrm{E}-3$ & 0.20 & 0.19908 & 0.20035 & 72 & 1.24 & 1.29 & 0.50 & 0.04 \\
\hline $4 \_3 \mathrm{a}$ & $1.50 \mathrm{E}-2$ & 0.05 & 0.05117 & $\ldots$ & 72 & 0.84 & 3.60 & 2.80 & 2.72 \\
\hline 4_3b & $9.82 \mathrm{E}-3$ & 0.08 & 0.08078 & 0.08168 & 72 & 2.61 & 3.59 & 2.79 & 0.91 \\
\hline $4 \_3 \mathrm{c}$ & $7.95 \mathrm{E}-3$ & 0.10 & 0.10214 & $\ldots$ & 72 & 2.63 & 3.60 & 2.80 & 0.88 \\
\hline $4 \_3 \mathrm{~d}$ & $5.36 \mathrm{E}-3$ & 0.15 & 0.15314 & $\ldots$ & 72 & 3.38 & 3.59 & 2.79 & 0.11 \\
\hline 4_3e & $4.04 \mathrm{E}-3$ & 0.20 & 0.20412 & $\ldots$ & 72 & 3.48 & 3.60 & 2.80 & 0.04 \\
\hline 4_4a & $1.85 \mathrm{E}-2$ & 0.05 & 0.05182 & $\ldots$ & 72 & 0.86 & 5.03 & 4.24 & 4.08 \\
\hline $4 \_4 \mathrm{~b}$ & $1.22 \mathrm{E}-2$ & 0.08 & 0.08029 & 0.08276 & 72 & 3.53 & 5.04 & 4.29 & 1.45 \\
\hline $4 \_4 \mathrm{c}$ & $9.85 \mathrm{E}-3$ & 0.10 & 0.10341 & $\ldots$ & 72 & 4.30 & 5.06 & 4.27 & 0.64 \\
\hline $4 \_4 \mathrm{~d}$ & $6.65 \mathrm{E}-3$ & 0.15 & 0.15512 & $\ldots$ & 72 & 4.82 & 5.06 & 4.27 & 0.10 \\
\hline $4 \_4 \mathrm{e}$ & $5.00 \mathrm{E}-3$ & 0.20 & 0.20681 & $\ldots$ & 72 & 4.93 & 5.05 & 4.26 & 0.01 \\
\hline
\end{tabular}



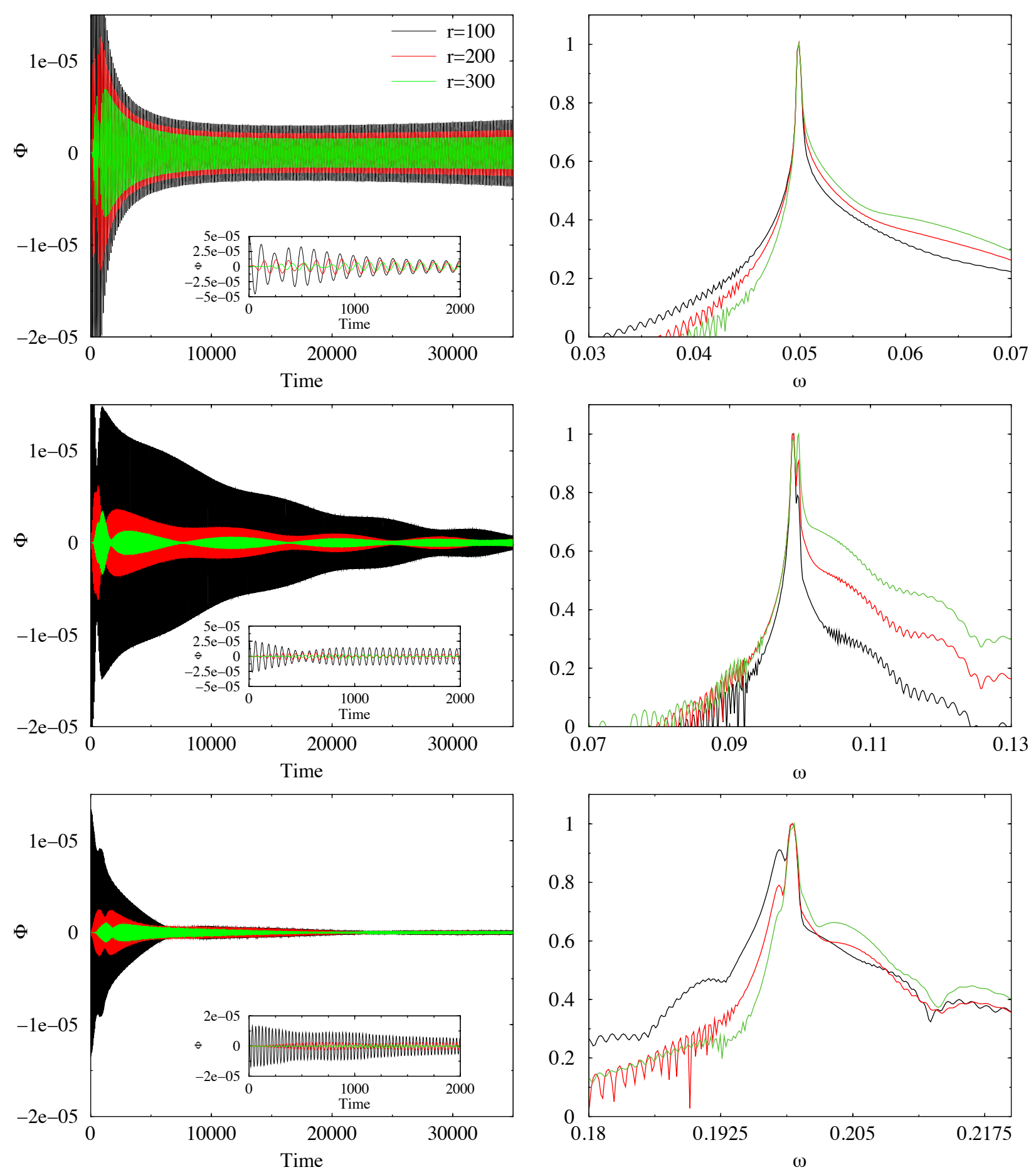

FIG. 4: Left column: Time evolution of the scalar field with initial energy $E_{0}=3.2 \times 10^{-5}$ for the TOV model 2 and three different scalar field masses $\mu=\{0.05,0.1,0.2\}$ (from top to bottom). The inset shows a magnified view of the initial 2000 units of time in the evolution. Right column: Fourier transform of the evolution of the scalar field. $\omega$ refers to the real part of the frequencies. The units in the vertical axis are arbitrary and are normalized by the amplitude of the fundamental mode. Each curve corresponds to a different extraction radius, as indicated in the legend of the top-left plot. 

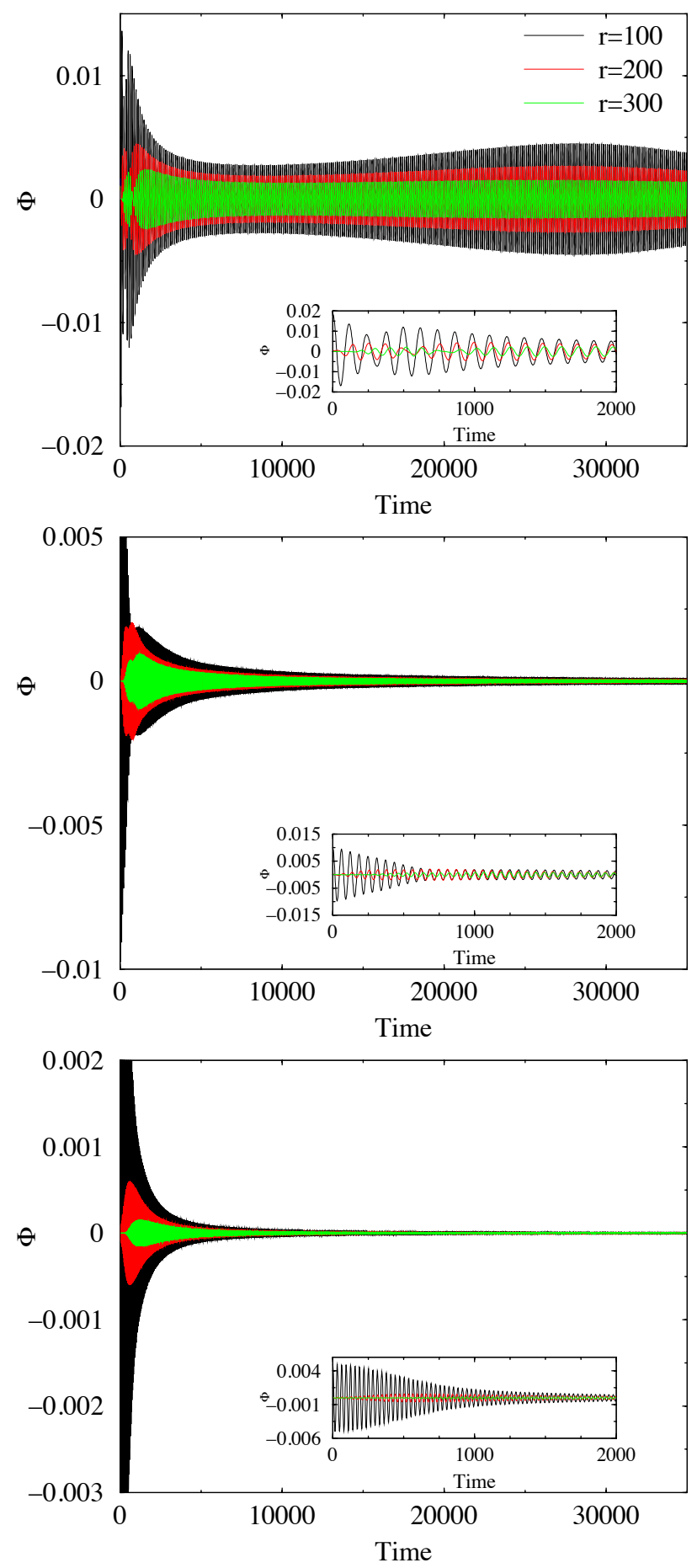

FIG. 5: Time evolution of the scalar field with initial energy $E_{0}=4.3$ for the TOV model 2 and three different scalar field masses $\mu=\{0.05,0.1,0.2\}$ (from top to bottom). The inset shows a magnified view of the initial 2000 units of time in the evolution.
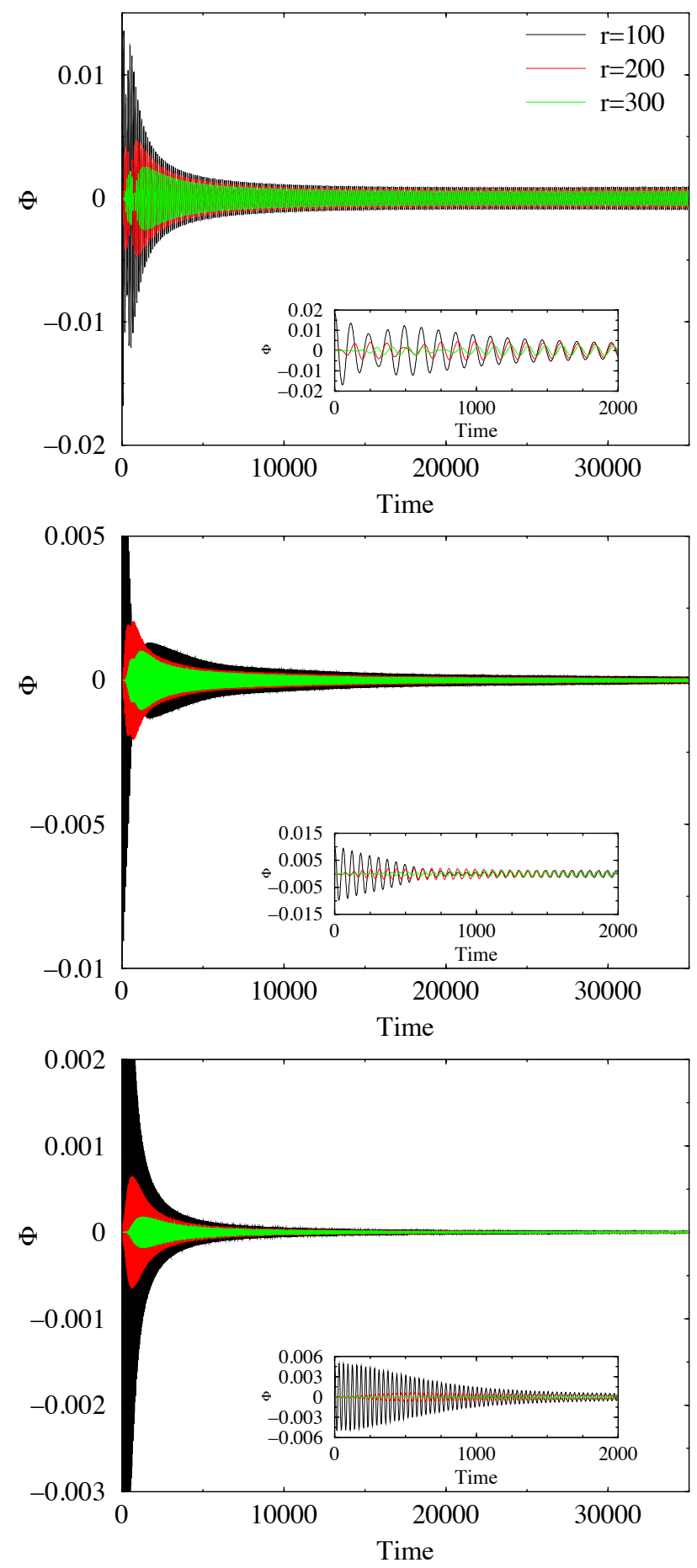

FIG. 6: Time evolution of the scalar field with initial energy $E_{0}=4.3$ for the TOV model 4 and three different scalar field masses $\mu=\{0.05,0.1,0.2\}$ (from top to bottom). The inset shows a magnified view of the initial 2000 units of time in the evolution. 


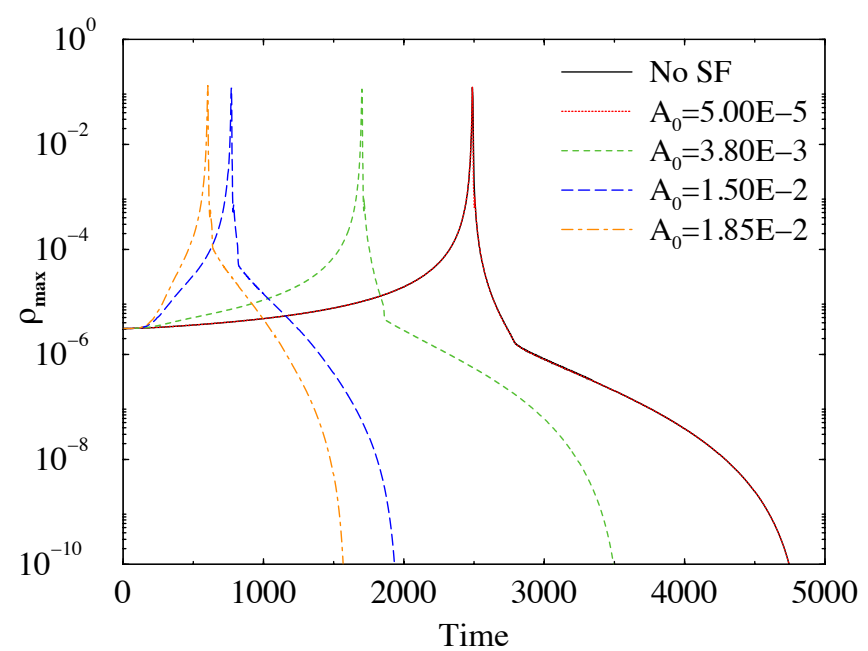

FIG. 7: Time evolution of the maximum (central) rest-mass density of the TOV model 1 for different initial values of the amplitude of the scalar field pulse with mass $\mu=0.05$. Notice that the black and red solid lines overlap.

alyze the results of the simulations by extracting a time series for the scalar field amplitude at a set of observation points located at fixed radii $r_{\text {ext }}$ (typically at three radii located at $r_{\text {ext }}=100,200$ and 300). To identify the frequencies at which the scalar field oscillates we perform a Fast Fourier transform after a given number of time steps and obtain the corresponding power spectrum. The very existence of these frequencies for the long-term simulations we perform is the main indicator we use to demonstrate the presence of quasi-bound states.

The main results of our simulations are summarized in Tables IIV] These four tables contain for each TOV model, the different choices for the scalar field mass $\mu$, the initial amplitude of the pulse $A_{0}$, the Arnowitt-DeserMisner (ADM) mass $M_{\mathrm{ADM}}$ of the whole system, defined as

$$
M_{\mathrm{ADM}}=-\frac{1}{2 \pi} \lim _{r \rightarrow \infty} \oint_{S} \partial_{j} \psi d S^{j},
$$

and the initial energy of the scalar field $E_{0}$

$$
E=\int_{2 M}^{\infty} \mathcal{E}^{\mathrm{SF}} d V
$$

where $\mathcal{E}^{\mathrm{SF}}$ is defined in Eq. 18. As the second-to-last column of each table shows, we vary the initial amplitude for the different $\mu$ in order to keep the initial scalar field energy constant for four different scenarios, from the test-field approximation to the non-linear regime where the scalar field is self-gravitating. This is presented as the four subdivisions of each table. The oscillations frequencies of the scalar field are reported in columns 4 and 5 for the fundamental mode and first overtone, respectively.

Figures 4,6 show the time evolution of the scalar field amplitude extracted at three different radii, $r_{\text {ext }}=$
$\{100,200,300\}$. In the left panels of Fig. 4 we plot the evolution for the initial scalar field energy $E_{0}=3.2 \times 10^{-5}$ for the TOV model 2 and for three scalar field masses, namely $\mu=\{0.05,0.1,0.2\}$ from top to bottom. The three panels show a moderately rapid decay of the amplitude of the oscillations of the scalar field. However, the field amplitudes by the end of the simulations have not totally vanished, especially for the $\mu=0.05$ model shown in the top panel. Correspondingly, the right panels of Fig. 4 present the power spectra, showing the well-defined oscillation frequencies of the quasi-stationary states. It is worth stressing that the values of the frequencies do not depend on the location of the observer.

Similar time evolution plots are displayed in figure 5 but, in this case, for the larger initial scalar field energy of our sample, $E_{0}=4.3$ (keeping the same values of $\mu$ and TOV model as in Fig. 4). The evolution for the same initial parameters corresponding to the TOV model 4 can be found in figure 6. The comparison among these time evolution plots shows that for the smallest scalar field mass, $\mu=0.05$, the differences between the evolutions for the different TOV models are more evident. The TOV model 2 shows an increase in the amplitude of the oscillations at the end of the simulation due to the presence of two close frequencies giving rise to a beating pattern, while TOV model 4 does not show this behavior. However, for larger scalar field masses, the differences in the time evolutions become qualitatively less important.

The power spectra obtained from the Fourier transforms of these time series show a set of distinct frequencies, exemplified in the right panels of Fig. 4 for a particular set of models. The frequency resolution is inversely proportional to the simulation time and hence the longer the runs the more accurate the frequencies. The presence of these frequencies confirms the existence of long-lived spherical quasi-bound states of scalar fields surrounding black holes even if these are formed dynamically through the gravitational collapse of spherical stars. During the non-linear evolution of the system, part of the scalar field survives the gravitational collapse and remains in the form of trapped states. These results extend the validity of our previous model, put forward in 32 and Paper 1 , in the linear and non-linear regimes respectively, to a significantly more dynamical scenario.

In columns 4 and 5 of Tables II [V] we show the frequencies $\omega$ of the scalar field oscillations. These frequencies refer only to the real part of the dominant modes and are labelled according to their strength on the power spectrum. For some models we are able to obtain several frequencies of oscillation. Tables II [V] also report the mass of the apparent horizon $(\mathrm{AH})$ at the end of the simulation $M_{\mathrm{AH}}$, defined as $M_{\mathrm{AH}}=\sqrt{\mathcal{A} / 16 \pi}$, where $\mathcal{A}$ is the area of the $\mathrm{AH}$, the time of collapse at which the $\mathrm{AH}$ is first found, $t_{\mathrm{col}}$, and the scalar field energy $E_{\text {final }}$ at the end of the simulation. In figure 7 we plot the time evolution of the central rest-mass density of the TOV model 1 for different values of the scalar field amplitude. This figure shows that the time at which the TOV collapses 
decreases monotonically when the initial scalar field amplitude increases. We note that $t_{\text {col }}$ for the case of the test-field scalar field energy $\left(A_{0}=5 \times 10^{-5}\right)$ coincides perfectly with the corresponding time for the case with no scalar field affecting the density evolution of the TOV star (as both lines overlap in the figure).

From Tables II [V] we see that the time of collapse shows a clear dependence with the initial energy of the scalar field $E_{0}$ only for the less compact TOV models of our sample, models 1 and 2 for which the values of the compactness parameter $M / R$ are 0.008 and 0.015 , respectively. This time becomes shorter the larger $E_{0}$. Model 3 , with $M / R=0.025$, has significantly shorter values of $t_{\text {col }}$ and, moreover, $t_{\text {col }}$ barely shows any dependence on $E_{0}$. Finally, for the TOV model 4 with $M / R=0.042$ the time of collapse is the shortest of the whole sample and constant, $t_{\mathrm{col}}=72$ irrespective of $E_{0}$. This result is explained by the fact that in this case the star collapses to form a black hole even before the scalar field pulse can reach the collapsing star. Therefore, the presence of the scalar field does not influence the collapse dynamics at all, even for the highest scalar field energies we consider in our sample.

The oscillation frequencies for the same scalar field parameters vary slightly from one TOV model to another due to the different initial mass of the black hole when it is formed. For most cases we find that the smaller the black hole mass the higher the frequencies. This trend, however, does not apply for the larger energy models, for which the frequencies are essentially identical for all the TOV stars. This behaviour might be simply a numerical artifact due to the lack of sufficient time resolution, as with increasing evolution time the frequencies could be better resolved and differences may appear.

In general our results show that when we consider large enough values of the scalar field mass $\mu$, a significantly large amount of the initial scalar field energy falls onto the black hole, and this amount increases with $\mu$ (see last columns of Tables II V . This behaviour is found for all initial scalar field energies considered. On the other hand, the amount of scalar field absorbed by the black hole changes depending on the TOV model, that is, on the initial mass of the black hole when it is formed. We find that it is slightly smaller for the models for which the TOV mass is smaller (and the compactness of the star is correspondingly larger). For the models with the largest mass $\mu$ and largest amplitude $A_{0}$, the energies at the end of the simulations are just a tiny fraction of the initial values (see last row of last columns of Tables II $[$ V). Even if long-lived quasi-stationary states can be found in such cases, their energies may be too small to question the actual survival of the scalar field. Nevertheless, for smaller values of $\mu$ this trend is reversed and only a small fraction of the initial energy of the scalar field is absorbed by the black hole.

Our results cannot be directly extrapolated to the case of a typical $10^{6} M_{\odot}$ SMS collapsing to a black hole. However, we may still put forward an educated guess of what

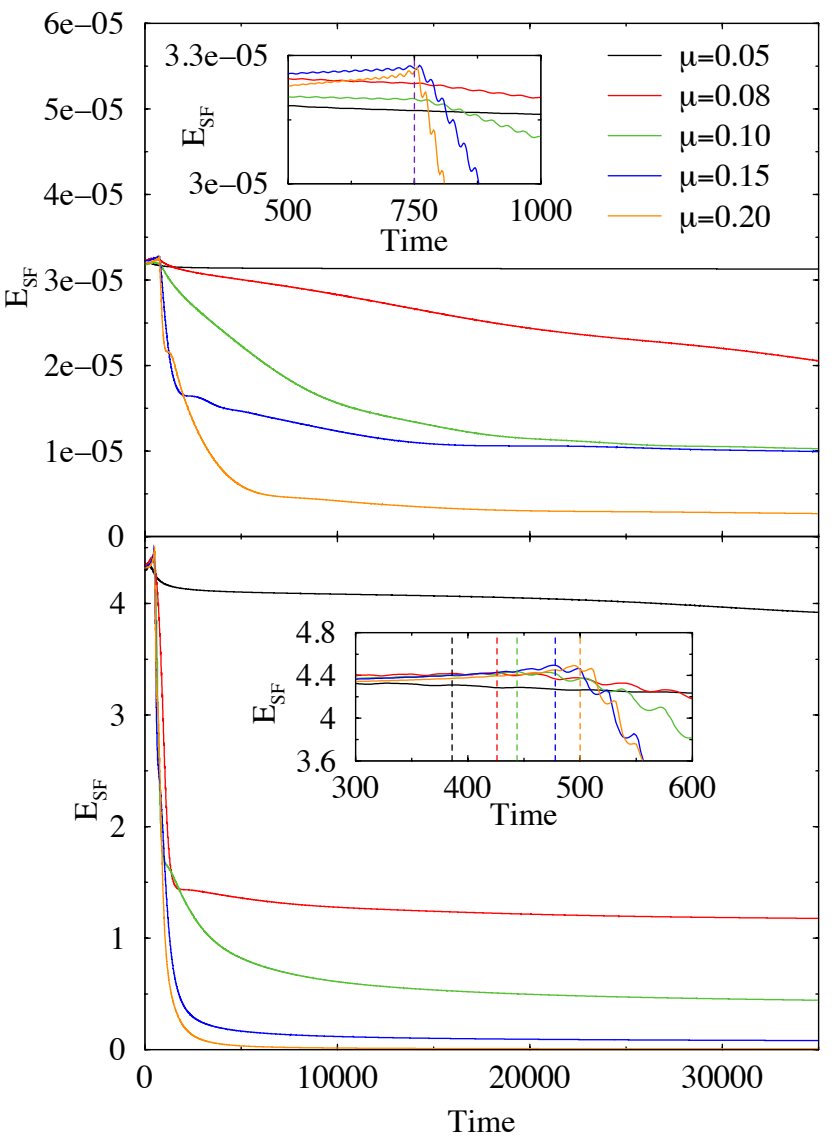

FIG. 8: Time evolutions of the scalar field energy from the initial value $E_{0}=3.2 \times 10^{-5}$ (top panel) and from the initial value $E_{0} \sim 4.3$ (bottom panel). The evolutions are shown for the TOV model 2 and for all scalar field masses $\mu=\{0.05,0.08,0.1,0.15,0.2\}$. The insets show magnified views around the time of collapse in the corresponding evolutions. The times at which the black holes are formed are displayed as vertical dashed lines. The different colors are related to the different scalar field masses as indicated in the legend of the top panel.

actually might happen. On the one hand, SMS are less compact $\left(M / R \sim 10^{-3}\right)$ than our TOV models and their gravity is almost Newtonian. Taking this into account, if the quasistationary states survive to the collapse of a more compact star, it could be expected that they will also persist after the collapse of a SMS. On the other hand, SMS collapse occurs at $t_{\text {col }} \sim 10^{5} \mathrm{~s}$, 9, which in our units is about $t_{\mathrm{col}} / M \sim 1000-40000$, depending on the compactness of the star. Our results yield $t_{\text {col }} / M \sim 90-1750$, and the simulations show that the scalar field survives at least for two to three orders of magnitude beyond the collapse timescale. In the context of a SMS, quasistationary states may then exist for long times.

The time evolution of the scalar field energy corre- 


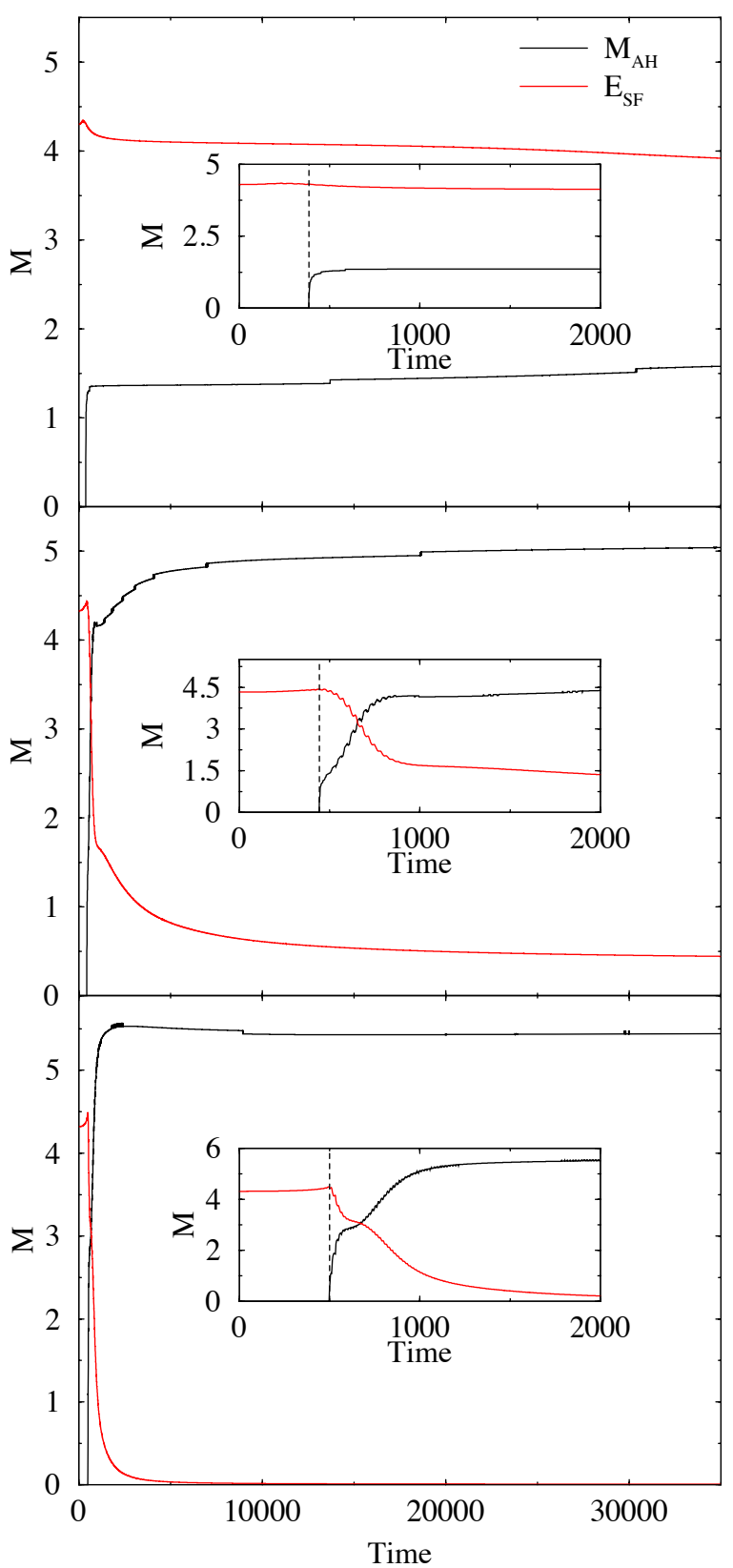

FIG. 9: Time evolution of the mass of the apparent horizon $M_{\mathrm{AH}}$ and of the scalar field energy for the initial $E_{0} \sim 4.3$ and the scalar field masses $\mu=\{0.05,0.1,0.2\}$ from top to bottom corresponding to the TOV model 2 . The inset shows a magnified view of initial 2000 in the evolution. The time at which the black hole is formed is displayed as a vertical dashed line.

sponding to the TOV model 2 and for all scalar field masses is shown in figure 8 . The top panel corresponds to $E_{0}=3.2 \times 10^{-5}$ and the bottom panel to $E_{0} \sim 4.3$ (the precise values are given in the second to last column of Table III, models 2.4a-2.4e). In the case of the test-field regime, the energy decay is smooth and varies monotoni-
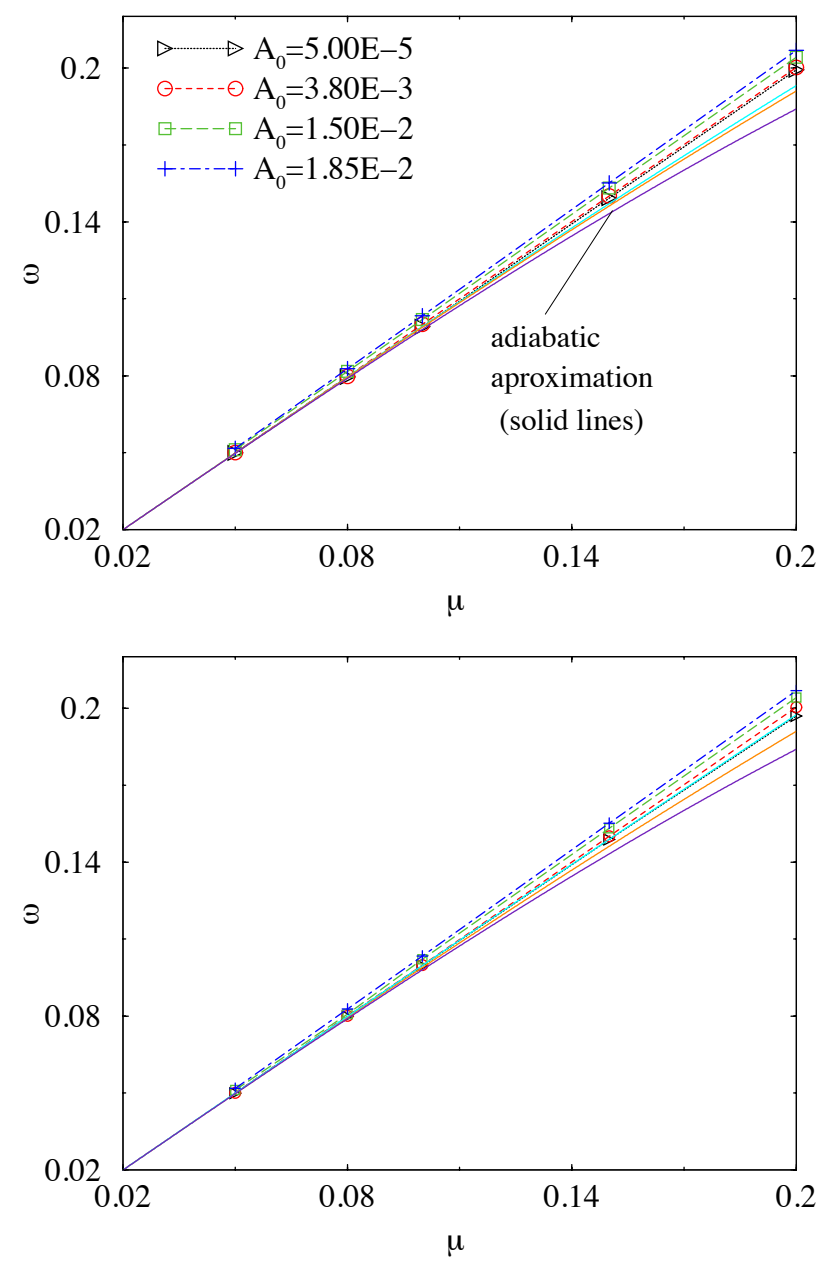

FIG. 10: Top panel: Frequency $\omega$ as a function of the scalar field mass $\mu$ for the models shown in Table III. The semianalytic relation given by Eq. (41) is also plotted for $M=$ $\{1.31,1.5,2.0\}$ (solid lines). The semi-analytic values for $M=$ 1.31 corresponds to the cyan line. Bottom panel: same as the top panel but for the models shown in Table $\mathrm{V}$. The semianalytic relation is plotted for $M=\{0.8,1.5,2.0\}$. The semianalytic values for $M=0.80$ corresponds to the cyan line.

cally with the increasing mass. In the non-linear regime, there is a significant leap (better seen in the inset of the bottom panel) in the amount of scalar field energy due to the initial accretion onto the black hole when it is formed. The decay of the energy after black hole formation (signalled by the dashed vertical lines in the figure) is in general fairly abrupt for all scalar field masses except for $\mu=0.05$. After the scalar field first interacts with the black hole, the energy reaches a phase during which the accretion rate is importantly reduced, allowing to the survival of the scalar field for long timescales (but again notice the drastic reduction in the scalar field energy for $E_{0} \sim 4.3$ and $\mu=0.15$ and $\mu=0.2$ ). This response of 
the system happens faster the larger the initial energy of the scalar field.

In Fig. 9 we plot the evolution of the scalar field energy $E_{\mathrm{SF}}$ (red curves) and the mass of the apparent horizon $M_{\mathrm{AH}}$ (black curves) for the TOV model 2 and the largest initial energy $\left(E_{0} \sim 4.3\right)$ for three scalar field masses, $\mu=\{0.05,0.1,0.2\}$ from top to bottom. The scalar field energy remains mostly constant until the time of collapse, when it begins to decrease (see the corresponding insets). The decrease is more significant and faster the larger the scalar field mass. Similarly, the mass of the black hole starts to increase once the apparent horizon (discontinually) appears, as a response to the accretion of the scalar field. It becomes clear from this figure that the amount of the scalar field lost in the process is absorbed by the black hole, becoming the only contribution to its growth when the fluid mass has been completely accreted by the black hole. The evolutions of both quantities are almost perfect mirror images from one another.

By using a matching technique, Furuhashi and Nambu showed analytically in 33 that in the limit $M \mu \ll 1$ the real part of the frequency of quasi-bound states, $\omega_{\mathrm{qbs}}$, depends on the mass parameter $\mu$ as

$$
\operatorname{Re}\left(\omega_{\mathrm{qbs}}\right) \approx \mu\left[1-\frac{1}{2}(M \mu)^{2}\right] .
$$

In Fig. 10 we show a plot of the frequencies of all the configurations of Table III and $\mathrm{V}$ as a function of $\mu$ (similar behaviour is found for the other models). Our numerical results are plotted with non-solid lines. We also consider in this plot the adiabatic approximation for the frequency given by Eq. (41) for different values of the mass of the black hole $M$. These analytic results are plotted with solid lines in the figure.

For the TOV model 4 (bottom panel), we find that for the smallest value of $A_{0}$, indicated in the legend in the top panel of the figure, the corresponding semi-analytic curve (black dotted line) is indistinguishable from the one of the test field approximation for $M=0.8$ (cyan solid line) showing consistency with the test-field approximation and the limit $M \mu \ll 1$. For the TOV model 2 (top panel), while the results are similar, for the larger scalar field mass the adiabatic approximation is not matched even in the test-field approximation for $M=1.31$ (cyan solid line). For all the models, we find that for greater values of $A_{0}$, the results from the non-linear approach not only deviate from the adiabatic approximation but they also follow the opposite trend: the frequency is greater than the frequency of the test-field limit. This discrepancy may be due to the violation of the condition $M \mu \ll 1$ used in the analytical approximation and due to a breakdown of the adiabatic approximation.

These results agree with our findings in Paper 1. When the initial amplitude is increased and the model is far from the test-field regime, the frequencies become higher. A similar trend is found when the scalar field mass is small, the frequencies becoming closer to those of the test-field regime. However, in this case we find that for the larger amplitudes the frequencies do not differ as much as in Paper 1 from the frequencies matching the adiabatic approximation. This may be due to the fact that depending on the initial profile and position of the scalar field pulse, there can be a variable amount of scalar field that ends up being part of the quasi-bound states.

\section{Comparison with a BH-scalar field system}

In order to further understand the influence of the collapse of the stars in the evolution of the scalar field, we also evolve an additional set of 12 models corresponding to purely Schwarzschild-like black hole spacetimes. These black holes have initial masses equal to those of the different TOV stars considered in the simulations we have just discussed. Likewise, the initial distributions of the scalar field clouds are the same as in the previous simulations. Therefore, the scalar field interacts from the beginning with an already existing black hole. This is the same situation we analyzed in Paper 1, only the initial black hole masses have now been adapted to match those of the TOV stars. We choose only three scalar field masses, $\mu=\{0.05,0.1,0.2\}$, and the largest value of the initial energy, $E_{0} \sim 4$.3. The grid resolution, time-step, and final evolution time remain unchanged. Table VI summarizes the results corresponding to this scenario.

In this set of simulations, as we showed in Paper 1, part of the scalar field is immediately accreted onto the black hole while part forms the quasi-bounds states. The determination of the oscillation frequencies of those states reveals that, for $\mu=0.1$ and 0.2 , some of the frequencies are equal to those of the collapsing star scenario. On the other hand, the final scalar field energy is, for the majority of models, smaller than that for the TOV setup but the difference is reduced either if the scalar field mass is increased or if the initial black hole mass is lowered. This discrepancy, most noticeable for TOV model 2, can be due to the fact that for the TOV a larger fraction of the scalar field may escape before the black hole forms and starts accreting. There is almost no difference between the two setups for TOV model 4 because, as mentioned before, the time of collapse is short enough to form a black hole before most of scalar field reaches the star. We also note that for the black hole models 1 and 2 and $\mu=0.05$ the amount of scalar field that falls onto the black hole is larger than for the other models and scalar field masses. As a result, the mass of the final black holes increases significantly and the oscillation frequencies change. The dynamics of the scalar field in the $\mathrm{BH}$ and TOV setups are markedly different for this particular set of models.

This is clearly visible in Fig. 11 where we plot the evolution of the scalar field energy for the initial parameters described in TableVI for both the TOV and BH models. As the mass of the scalar field increases from $\mu=0.05$ (left column of panels) to $\mu=0.2$ (right column of panels) the evolution of the energy becomes progressively 
TABLE VI: Same as Tables II V but for evolutions of scalar fields in spacetimes containing initially a Schwarzschild black hole. Only the fundamental frequency is shown.

\begin{tabular}{|c|c|c|c|c|c|c|c|c|}
\hline Model & $A_{0}$ & $\mu$ & $\omega$ & $\left(M_{\mathrm{AH}}\right)_{0}$ & $\left(M_{\mathrm{AH}}\right)_{\mathrm{final}}$ & $M_{\mathrm{ADM}}$ & $E_{0}$ & $E_{\text {final }}$ \\
\hline 1_4a_bh & $1.85 \mathrm{E}-2$ & 0.05 & 0.05116 & 1.43 & 3.70 & 5.73 & 4.31 & 1.88 \\
\hline 1_4c_bh & $9.85 \mathrm{E}-3$ & 0.10 & 0.10341 & 1.43 & 5.32 & 5.75 & 4.34 & 0.31 \\
\hline 1_4e_bh & $5.00 \mathrm{E}-3$ & 0.20 & 0.20681 & 1.43 & 5.62 & 5.75 & 4.33 & 0.007 \\
\hline 2_4a_bh & $1.85 \mathrm{E}-2$ & 0.05 & 0.05170 & 1.31 & 2.67 & 5.59 & 4.30 & 2.88 \\
\hline 2_4c_bh & $9.85 \mathrm{E}-3$ & 0.10 & 0.10341 & 1.31 & 5.18 & 5.62 & 4.33 & 0.34 \\
\hline 2_4e_bh & $5.00 \mathrm{E}-3$ & 0.20 & 0.20681 & 1.31 & 5.44 & 5.61 & 4.32 & 0.008 \\
\hline 3_4a_bh & $1.85 \mathrm{E}-2$ & 0.05 & 0.05170 & 1.10 & 1.34 & 5.36 & 4.28 & 3.93 \\
\hline 3_4c_bh & $9.85 \mathrm{E}-3$ & 0.10 & 0.10341 & 1.10 & 4.87 & 5.39 & 4.30 & 0.41 \\
\hline 3_4e_bh & $5.00 \mathrm{E}-3$ & 0.20 & 0.20681 & 1.10 & 5.26 & 5.38 & 4.29 & 0.01 \\
\hline 4_4a_bh & $1.85 \mathrm{E}-2$ & 0.05 & 0.05188 & 0.80 & 0.86 & 5.04 & 4.24 & 4.08 \\
\hline 4_4c_bh & $9.85 \mathrm{E}-3$ & 0.10 & 0.10341 & 0.80 & 4.32 & 5.06 & 4.27 & 0.64 \\
\hline 4_4e_bh & $5.00 \mathrm{E}-3$ & 0.20 & 0.20681 & 0.80 & 4.93 & 5.05 & 4.26 & 0.01 \\
\hline
\end{tabular}

similar, and the presence of a black hole from the start or at later times is unimportant for the dynamics. The same trend is found as the initial black hole mass or TOV mass is reduced (see bottom row of panels in Fig. 11). In this case the dynamics also becomes identical irrespective of the scalar field mass. The major differences are found in the upper left corner of the composite of panels in Fig. 11, i.e. when $\mu$ is the smallest and the black hole and TOV initial masses are largest. In any event, the influence of the gravitational collapse on the dynamics of the scalar field and on the eventual presence of quasibound states is not as potentially threatening as we might have expected before conducting this research. The initial (rapid) phase of accretion by an already formed black hole seems to overcome any dynamical effect from the collapse of the star which might have affected the survival of the scalar field, even for the smaller scalar field masses and the larger initial black hole masses considered in our study, where different evolutions are observed.

\section{CONCLUSIONS}

In this paper we have discussed the feasibility of the existence of quasi-stationary states of self-gravitating scalar fields in the form of clouds (or hairy "wigs") around collapsing, black-hole-forming, stars. For our analysis we have solved numerically the Einstein-Klein-Gordon coupled system of equations further coupled to the Euler equations of general relativistic hydrodynamics. Our numerical approach has been limited to spherical symmetry and has been based on spherical coordinates and a PIRK numerical scheme for the time update. This research extends our previous work where the same issue was investigated for black holes already existing at $t=0$ to a much more dynamical scenario where the black holes are formed as the result of the gravitational collapse of unstable (polytropic) stars.
In our non-linear study we have performed a large number of (second-order) accurate and long-term stable simulations of dynamical non-rotating collapsing stars, with an EOS corresponding to SMSs, surrounded by selfgravitating scalar fields. The models of our sample have been suitably parameterized to span a broad range of scalar field cases, from the test-field regime to the fully non-linear regime, along with different initial masses for the star. We have found that in the case of highly dynamical spacetimes there are states which closely resemble the quasi-bound states in the test-field approximation of [20, 32, surviving the stellar collapse and black hole formation.

By performing a Fourier transform of the time series of our numerical data we have been able to characterize the scalar field states by their distinctive oscillation frequencies. It has been found that the scalar field oscillates with different well-defined frequencies, and that its time evolution produces in most of our models a characteristic beating pattern due to the non-linear combination of two oscillations with close frequencies. Such scalar field oscillations may have important imprints in a number of astrophysical scenarios such as gravitational wave astronomy. In particular [18, 34] have shown recently that the frequency of oscillation of the scalar field leaves a distinctive signature in the late time behaviour of the gravitational wave signal.

We have also compared the evolutions of the scalar field in collapsing, black-hole-forming spacetimes with spacetimes that already contain a non-rotating black hole. This comparison has provided a better understanding of the influence of the stellar collapse on the scalar field dynamics. Our results have shown that the differences are mainly due to the initial (or lack of) accretion phase onto the black hole that contributes to the growth of its mass and to the different dynamics, in the case of selfgravitating scalar fields.

Furthermore, our results indicate that for certain val- 

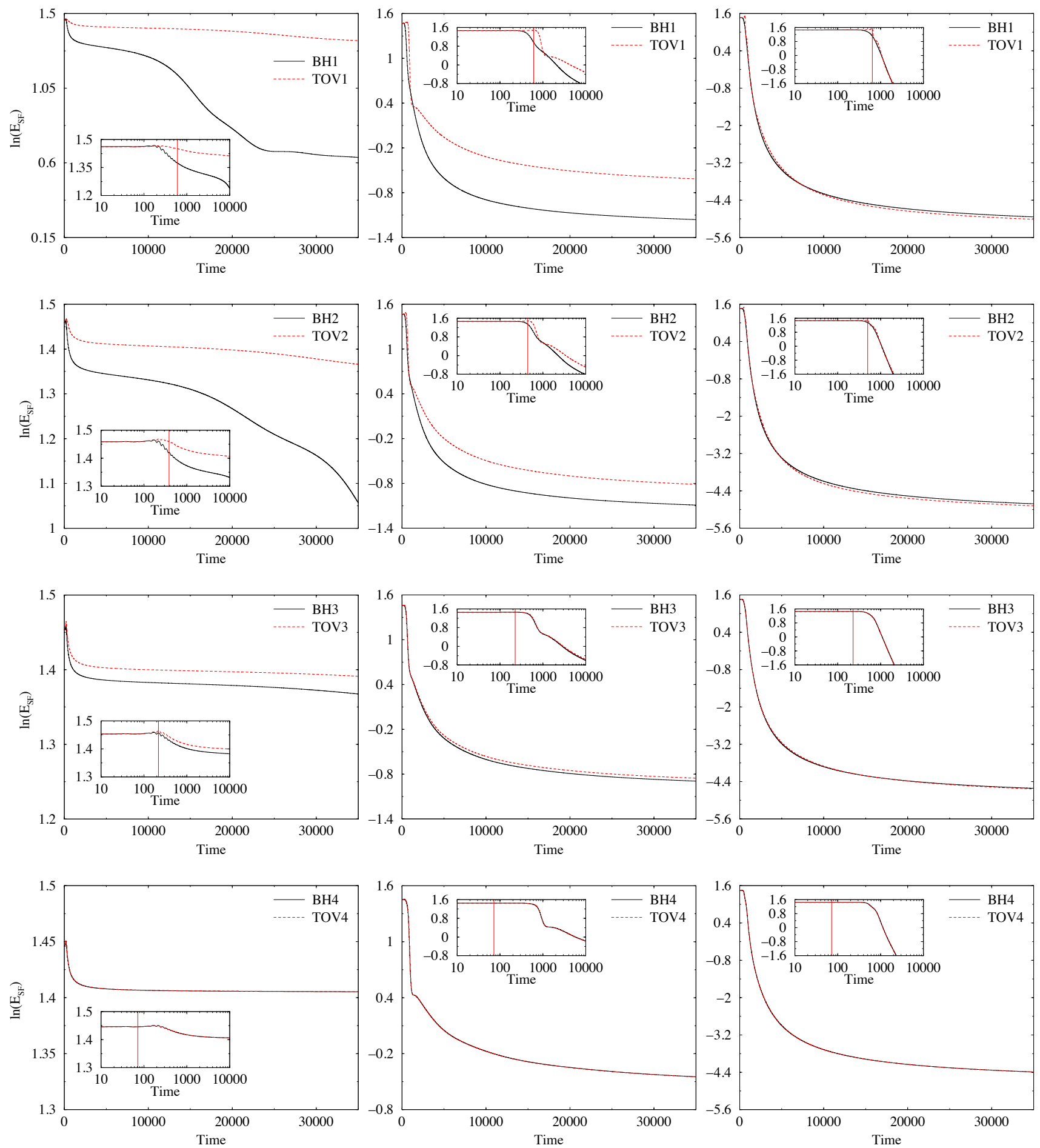

FIG. 11: Comparison of the time evolution of the scalar field energy for the collapsing TOV star models and for Schwarzschild black holes with the same initial mass. The initial energy density is $E_{0} \sim 4.3$. The insets show magnified views in logarithm scale of the initial 10000 units of time in the evolution. Left column: $\mu=0.05$. Middle column: $\mu=0.1$. Right column: $\mu=0.2$. 
ues of $\mu$, the formation of the black hole during the collapse of the star enhances the chances of the scalar field to remain in a quasi-bound state. This result may have important implications for models of black hole formation together with dark matter halos in the early epochs of the Universe. For instance, large galaxies in the scalar field model can already be formed at high redshift 35 .

The collapse of the $\sim 1 M_{\odot}$ stars considered in this work and the subsequent formation of the black holes modify the dynamics of the scalar field, but for the range of TOV masses this type of events has very short duration. While our results cannot be directly extrapolated to the case of a typical $10^{6} M_{\odot}$ SMS collapsing to a black hole, it could perhaps be expected, on the basis of their smaller compactness and almost Newtonian gravity, that quasistationary states might still persist after the collapse of a SMS star. Actual numerical simulations are however necessary to validate the viability of this scenario and the survival of quasi-bound states of scalar fields around $\mathrm{SMBH}$ for cosmological timescales. In addition to such simulations it is worth to study the evolution of scalar fields around accreting black holes during significantly longer evolutionary times than those attempted in [17], a task we defer for the near future.

Finally, we note that the dynamical scenario presented here can also be generalized to rotating stars where the final state may be a rotating Kerr black hole surrounded by a scalar field cloud yielding a hairy black hole solution [36, 37]. It has been argued that these solutions may act as dynamical attractors in a highly dynamical process [38] and thus simulations as the ones performed here would be able to describe their formation.

\section{Acknowledgements}

PM thanks Ewald Müller for comments on the manuscript. This work has been supported by the Spanish MINECO (AYA2013-40979-P), by the Generalitat Valenciana (PROMETEOII-2014-069), by the CONACyT-México, and by the Max Planck Institute for Astrophysics. The computations have been performed at the Servei d'Informàtica de la Universitat de València.
[1] D. J. Mortlock, S. J. Warren, B. P. Venemans, M. Patel, P. C. Hewett, R. G. McMahon, C. Simpson, T. Theuns, E. A. Gonzales-Solares, A. Adamson, et al., Nature 474, 616 (2011), ISSN 0028-0836, 10.1038/nature10159.

[2] B. P. Venemans, E. Bañados, R. Decarli, E. P. Farina, F. Walter, K. C. Chambers, X. Fan, H.-W. Rix, E. Schlafly, R. G. McMahon, et al., The Astrophysical Journal Letters 801, L11 (2015), URL http://stacks. iop.org/2041-8205/801/i=1/a=L11

[3] V. Bromm and A. Loeb, Astrophys. J. 596, 34 (2003), astro-ph/0212400.

[4] Z. Haiman and A. Loeb, Astrophys. J. 552, 459 (2001).

[5] M. C. Begelman, M. Volonteri, and M. J. Rees, Mon.Not.Roy.Astron.Soc. 370, 289 (2006), astro$\mathrm{ph} / 0602363$.

[6] M. C. Begelman, Mon.Not.Roy.Astron.Soc. 402, 673 (2010), 0910.4398.

[7] L. Mayer, S. Kazantzidis, A. Escala, and S. Callegari, Nature (London) 466, 1082 (2010), 0912.4262.

[8] M. Shibata and S. L. Shapiro, Astrophys. J. 572, L39 (2002), astro-ph/0205091.

[9] P. J. Montero, H.-T. Janka, and E. Müller, Astrophys. J. 749, 37 (2012), 1108.3090.

[10] J. E. Lidsey, A. R. Liddle, E. W. Kolb, E. J. Copeland, T. Barreiro, et al., Rev.Mod.Phys. 69, 373 (1997), astroph/9508078.

[11] T. Padmanabhan, Phys.Rept. 380, 235 (2003), hepth/0212290.

[12] V. Sahni and L.-M. Wang, Phys.Rev. D62, 103517 (2000), astro-ph/9910097.

[13] W. Hu, R. Barkana, and A. Gruzinov, Phys. Rev. Lett. 85, 1158 (2000), astro-ph/0003365.

[14] T. Matos and L. A. Urena-Lopez, Class. Quant. Grav. 17, L75 (2000), astro-ph/0004332.

[15] T. Matos and L. A. Urena-Lopez, Phys.Rev. D63,
063506 (2001), astro-ph/0006024.

[16] J. Barranco, A. Bernal, J. C. Degollado, A. Diez-Tejedor, M. Megevand, et al., Phys.Rev. D84, 083008 (2011), 1108.0931.

[17] N. Sanchis-Gual, J. C. Degollado, P. J. Montero, and J. A. Font, Phys. Rev. D 91, 043005 (2015), URL http: //link.aps.org/doi/10.1103/PhysRevD.91.043005.

[18] H. Witek, V. Cardoso, A. Ishibashi, and U. Sperhake, Phys.Rev. D87, 043513 (2013), 1212.0551.

[19] J. Barranco, A. Bernal, J. C. Degollado, A. Diez-Tejedor, M. Megevand, et al. (In preparation).

[20] J. Barranco, A. Bernal, J. C. Degollado, A. Diez-Tejedor, M. Megevand, et al., Phys.Rev. D89, 083006 (2014), 1312.5808.

[21] P. J. Montero and I. Cordero-Carrion, Phys.Rev. D85, 124037 (2012), 1204.5377.

[22] N. Sanchis-Gual, P. J. Montero, J. A. Font, E. Müller, and T. W. Baumgarte, Phys. Rev. D 89, 104033 (2014), URL http://link.aps.org/doi/10.1103/PhysRevD. 89.104033

[23] T. W. Baumgarte and S. L. Shapiro, Phys. Rev. D 59, 024007 (1998).

[24] M. Shibata and T. Nakamura, Phys. Rev. D 52, 5428 (1995).

[25] M. Alcubierre and M. D. Mendez, Gen.Rel.Grav. 43, 2769 (2011), 1010.4013.

[26] C. Bona, J. Massó, E. Seidel, and J. Stela, Phys. Rev. D 56, 3405 (1997), URL http://link. aps .org/doi/10. 1103/PhysRevD.56.3405

[27] M. Alcubierre, B. Brügmann, P. Diener, M. Koppitz, D. Pollney, E. Seidel, and R. Takahashi, Phys. Rev. D 67, 084023 (2003), URL http://link.aps .org/doi/10. 1103/PhysRevD.67.084023

[28] F. Banyuls, J. A. Font, J. M. Ibánez, J. M. Martí, and J. A. Miralles, Astrophys. J. 476, 221 (1997). 
[29] I. Cordero-Carrión and P. Cerdá-Durán, ArXiv e-prints (2012), 1211.5930.

[30] I. Cordero-Carrión and P. Cerdá-Durán, Advances in Differential Equations and Applications, SEMA SIMAI Springer Series Vol. 4 (Springer International Publishing Switzerland, Switzerland, 2014).

[31] N. Stergioulas and J. L. Friedmann, ApJ 444, 306 (1995).

[32] J. Barranco, A. Bernal, J. C. Degollado, A. Diez-Tejedor, M. Megevand, et al., Phys.Rev.Lett. 109, 081102 (2012), 1207.2153.

[33] H. Furuhashi and Y. Nambu, Prog.Theor.Phys. 112, 983 (2004), gr-qc/0402037.
[34] J. C. Degollado and C. A. R. Herdeiro, Phys.Rev. D90, 065019 (2014), 1408.2589.

[35] J. Magaña, T. Matos, A. Suárez, and F. J. SanchezSalcedo, JCAP 10 (2012).

[36] C. A. R. Herdeiro and E. Radu, Phys.Rev.Lett. 112, 221101 (2014), 1403.2757.

[37] C. Herdeiro and E. Radu, Class.Quant.Grav. 32, 144001 (2015), 1501.04319.

[38] C. L. Benone, L. C. B. Crispino, C. Herdeiro, and E. Radu, Phys.Rev. D90, 104024 (2014), 1409.1593. 